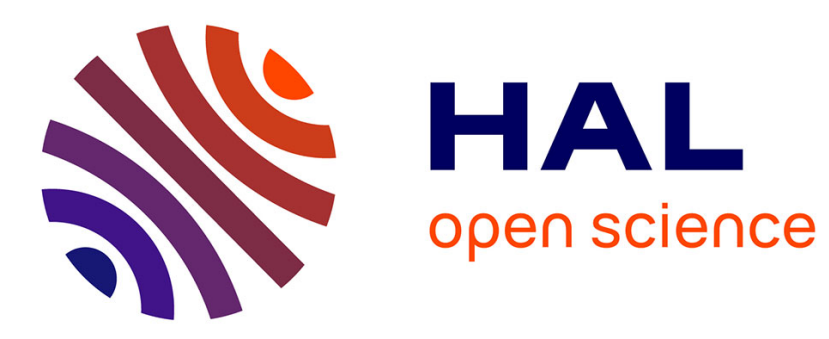

\title{
Traveling Fronts in Dissipative Granular Chains and Nonlinear Lattices
}

Guillaume James

\section{To cite this version:}

Guillaume James. Traveling Fronts in Dissipative Granular Chains and Nonlinear Lattices. Nonlinearity, 2021, 34 (3), pp.1758-1790. 10.1088/1361-6544/abdbbe . hal-02906265

\section{HAL Id: hal-02906265 \\ https://hal.science/hal-02906265}

Submitted on 24 Jul 2020

HAL is a multi-disciplinary open access archive for the deposit and dissemination of scientific research documents, whether they are published or not. The documents may come from teaching and research institutions in France or abroad, or from public or private research centers.
L'archive ouverte pluridisciplinaire HAL, est destinée au dépôt et à la diffusion de documents scientifiques de niveau recherche, publiés ou non, émanant des établissements d'enseignement et de recherche français ou étrangers, des laboratoires publics ou privés. 


\title{
TRAVELING FRONTS IN DISSIPATIVE GRANULAR CHAINS AND NONLINEAR LATTICES
}

\author{
GUILLAUME JAMES ${ }^{1,2}$
}

\begin{abstract}
We consider an infinite chain of particles with nonlinear elastic and dissipative nearest neighbors interactions. Assuming the existence of a traveling front between uniformly compressed (or stretched) states, we obtain jump conditions relating the wave speed and limiting particle velocities to the relative displacements at infinity. Using this result, we characterize compression fronts in chains of touching beads, for viscoelastic contact laws that include a nonlinear elastic force (generalized Hertz contact) and viscous dissipation. We compute compression fronts numerically for the generalized KuwabaraKono model in which the viscous contact force is proportional to the derivative of the elastic force, without precompression of the chain. Steady fronts are obtained both as the end result of the compression of one end of the chain and using a shooting method which provides numerically exact traveling waves. Depending on the magnitudes of contact damping and strain applied downstream, we obtain either overdamped (monotonic) or underdamped (oscillatory) compression fronts. To explain this transition and approximate the front profiles, we consider a continuum limit valid when the exponent of the contact nonlinearity is close to (and above) unity. Using multiscale expansions, we formally derive two different amplitude equations for long waves, a Burgers equation with logarithmic nonlinearity, and a logarithmic Korteweg-de Vries (KdV)-Burgers equation for small contact damping. Both models possess traveling front solutions that are in good agreement with the front profiles computed numerically in the granular chain. The analysis of the logarithmic KdV-Burgers equation allows one to approximate the critical damping corresponding to the transition from underdamped to overdamped fronts.
\end{abstract}

\section{INTRODUCTION}

Modeling the response of granular media to dynamic loading is important for many engineering applications, e.g. the dynamics of ballasted railway tracks [46], impact mitigation $[41,17,8]$ and acoustic lensing or filtering based on granular metamaterials [44, 53]. In order to understand the energy redistribution induced by multiple impacts and to predict post-impact velocities, it is fundamental to analyze wave effects $[15,24,43]$ such as the propagation of solitary waves in chains of beads, see e.g. $[40,49,9,31,27,30]$ and references therein. In this work, we examine another important class of waves consisting of compression fronts propagating in an homogeneous granular chain. These waves travel with a constant velocity and a constant shape, with the chain (asymptotically) at rest ahead of the shock and uniformly compressed at the rear. They can be typically generated by compressing the chain with a piston at constant velocity [39]. A correct modeling of this phenomenon requires to take contact damping into account $[19,36]$. Depending on the amount of dissipation induced by the material, oscillatory (underdamped) or monotonic (overdamped) shock profiles can be observed experimentally [40] (section 1.8 .7 p.80).

In this work, we consider a chain of identical beads described by the dynamical equations :

$$
m \ddot{x}_{n}=F\left(x_{n-1}-x_{n}, \dot{x}_{n-1}-\dot{x}_{n}\right)-F\left(x_{n}-x_{n+1}, \dot{x}_{n}-\dot{x}_{n+1}\right),
$$

where $x_{n}$ is the displacement of the center of the $n^{\text {th }}$ element from a reference position (corresponding to an uncompressed chain of touching beads), $m$ is the mass of beads, the overdots denote time

Date: July 23, 2020.

Key words and phrases. Granular chains, generalized Hertz contact, viscoelastic contact law, dissipative lattices, multiple impacts, traveling fronts, jump conditions, multiscale expansions, logarithmic Burgers and KdV-Burgers equations, numerical computation of wave profiles. 
derivatives and $F$ is a general contact force accounting for nonlinear elasticity and viscous friction [37]. Considering two adjacent beads compressed with a small relative displacement $\delta(t)$, we have

$$
F(\delta, \dot{\delta})=k\left(\delta_{+}^{\alpha}+\gamma_{0} \frac{d}{d t} \delta_{+}^{\beta}\right)
$$

where $\delta_{+}=\max (\delta, 0)$ (no force is present when beads are not in contact) and $k, \gamma_{0}$ are positive constants. The first term at the right side of (2) corresponds to the elastic component of the (generalized) Hertz contact force. The case of contacting spheres (or smooth non-conforming surfaces) leads to a strongly nonlinear contact force with $\alpha=3 / 2$, and the nonlinear stiffness $k$ depends on the radii of curvature and elastic coefficients of the balls [32]. Moreover, granular chains involving different orders of nonlinearity $\alpha>1$ have recently attracted much attention, see $[48,52]$ and references therein. In particular, experimental and numerical studies on solitary wave propagation have been performed with chains of hollow spherical particles of different width [42] and chains of cylinders [34], leading to different values $\alpha$ in the range $1.15 \leq \alpha \leq 1.5$ (see also [51] for other systems with $\alpha$ close to unity). The second term at the right side of (2) accounts for viscoelastic damping at small impact velocities (see [7] for a review of these contact laws).

In the works $[19,36]$, compression fronts are studied in the case $\beta=1$ (Hertzian spring with linear dashpot). With a precompression applied to the chain, classical multiscale expansions are performed to capture the leading order nonlinear, dispersive and dissipative features of small amplitude long waves. When a small contact damping is considered, the KdV-Burgers equation [33] is used to approximate traveling fronts and explain the transition from overdamped to underdamped profiles $[19,36]$ (see also $[12,23,3]$ for an application of this technique to other interactions between particles). In particular, the underdamped regime is observed when the impact velocity is large enough [36]. This approach is extended in [19] to the case without precompression, where a formal continuum limit leads to a second order differential equation with nonlinear damping for the front profile.

In the present paper, we study compression fronts for the generalized Kuwabara-Kono (KK) model with $\beta=\alpha$, and in particular for the standard KK model corresponding to $\beta=\alpha=3 / 2$ [35]. The KK model has the advantage of being derived from continuum mechanics (see $[6,18]$ and references therein) and $\gamma_{0}$ is determined by the elastic and viscous coefficients of the material. Moreover, it was shown in [30] that the KK model approximates the numerical dissipation of a class of first order RungeKutta schemes (including the $\theta$ method) used to integrate nondissipative granular chains. Consequently, the KK model can be also useful in the context of nondissipative Hertzian chains, in order to evaluate the effect of numerical damping on the dynamics of nonstationary shock waves (a problem suggested in [39]).

In section 2, we relate the asymptotic downstream bead compression and velocity to the speed of the shock wave in an infinite granular chain. A difference with previous results $[19,36]$ is that we do not resort to a continuum approximation at this stage. Moreover, we obtain a result for system (1) with $F$ deriving from a general elastic interaction potential and an arbitrary convex dissipation potential. Under the assumption that a traveling front exists, we relate the wave speed and velocity jump between $\pm \infty$ to the compression (or dilation) of the chain at $\pm \infty$. Similar results were obtained in [3] when the interaction force admits a nonlinear strictly convex elastic component and a linear dissipation, and in [21] for conservative systems where the interaction force has a turning point. Note that traveling fronts connecting uniformly compressed (or stretched) states do not exist in nondissipative chains with either strictly concave or convex interaction force $[3,21]$, which is the case for the generalized Hertz potential in the compressive domain when $\alpha \neq 1$.

In section 3, we show numerically that fronts can be generated in the KK model by applying a constant pressure at one end of a chain initially at rest. The front profile is underdamped or overdamped depending whether the dissipation constant $\gamma_{0}$ lies below or above a critical value proportional to $v_{\mathrm{p}}(1-\alpha) /(1+\alpha)$, where $v_{\mathrm{p}}$ denotes the asymptotic particle velocity after the shock. Consequently, for 
a given granular chain with KK contact law, the underdamped regime occurs when the downstream velocity $v_{\mathrm{p}}$ is small, unlike for $\beta=1$ and $\alpha>1[19,36]$.

In order to gain analytical insights into the existence and qualitative properties of fronts in the absence of precompression of the chain, we introduce suitable continuum limits of the generalized KK model. For this purpose, we perform a multiscale expansion of the slowly varying wave profiles when $\alpha$ is close to (and above) unity. Indeed, in the strongly nonlinear system (1)-(2), the width of localized traveling waves does not diverge in the small amplitude limit when $\alpha$ is fixed, but it diverges when $\alpha$ goes to unity, as such waveforms do not persist in the linear chain [38,3]. The limit $\alpha \approx 1$ was introduced in [10] to study solitary waves in nondissipative granular chains without precompression, and generalized in [27] to the nonstationary case. It yields a logarithmic nonlinearity $\delta_{+}\left(\ln \delta_{+}\right)$in the first order expansion of (2), which leads to a logarithmic KdV equation [47] for the wave profile and strongly localized (nearly Gaussian) solitary waves (see also [28, 29] for similar results for breather solutions and their connection to the logarithmic NLS equation [4]). Here we formally derive two different amplitude equations for long waves depending on the parameter regime. In section 4, we derive a logarithmic Burgers equation [47] that accounts for nonlinear and dissipative effects but neglects dispersion. We check numerically that this amplitude equation allows to approximate over long times the evolution of some localized initial conditions in the compressive regions (this class of models does not capture gap openings). When contact damping is small, dispersive effects must be taken into account, which leads to a logarithmic KdV-Burgers equation (section 5). This limit allows one to approximate the critical damping that corresponds to the transition from underdamped to overdamped fronts (this critical value becomes small when $\alpha \approx 1$ ). Both models possess traveling front solutions that provide good approximations to the steady wave profiles in the original lattice. The numerical computation of traveling fronts in the granular chain is performed in section 6 , using a shooting method combined with the Gauss-Newton method.

\section{TRAVELING FRONTS IN DISSIPATIVE LATTICES}

In this section we study a general class of dissipative one-dimensional lattices (1) that incorporates the granular chain with contact law (2). We consider general interaction forces between nearest neighbors taking the form

$$
F(\delta, \dot{\delta})=-V^{\prime}(-\delta)-\frac{d}{d t} W^{\prime}(-\delta)
$$

where the functions $V, W$ are sufficiently smooth. The potential $W$ is assumed convex and corresponds to dissipative forces, while the potential $V$ accounts for conservative forces. For example, the contact law (2) corresponds to fixing

$$
V(r)=\frac{k}{\alpha+1}(-r)_{+}^{\alpha+1}, \quad W(r)=\frac{\gamma_{0} k}{\beta+1}(-r)_{+}^{\beta+1} .
$$

The conservative case $W=0$ corresponds to a Fermi-Pasta-Ulam (FPU) lattice [16].

We shall derive jump conditions for traveling fronts relating the asymptotic states of the system at $\pm \infty$ and the wave velocity, in analogy with the Rankine-Hugoniot conditions for hyperbolic systems. These jump conditions have been obtained in [3] for linear dissipative forces (i.e. considering a quadratic potential $W$ ) and assuming $V^{(3)}<0$ for conservative forces (see also [21] for the analysis of the conservative case). Our computations follow the ideas of [3] although we consider more general conservative and dissipative forces and proceed slightly differently.

Let us start by introducing some notations and the dynamical equations. Given sequences $x=$ $\left(x_{n}\right)_{n \in \mathbb{Z}}, y=\left(y_{n}\right)_{n \in \mathbb{Z}}$ and a real function $f$, we denote by $f(x)$ the sequence $\left(f\left(x_{n}\right)\right)_{n \in \mathbb{Z}}$ and by $x y$ the sequence $\left(x_{n} y_{n}\right)_{n \in \mathbb{Z}}$. Moreover, $\left(\delta^{ \pm} x\right)_{n}= \pm\left(x_{n \pm 1}-x_{n}\right)$ denote the usual forward and backward differences. We consider an infinite chain of identical particles described by the model (1)-(3). The 
dynamical equations can be written in condensed form :

$$
m \ddot{x}=\delta^{-}\left(V^{\prime}\left(\delta^{+} x\right)+\frac{d}{d t} W^{\prime}\left(\delta^{+} x\right)\right),
$$

where $x(t)=\left(x_{n}(t)\right)_{n \in \mathbb{Z}}$ and we fix $m=1$ for convenience.

The dissipative character of the model can be illustrated by considering solutions with finite energy $H=\sum_{n \in \mathbb{Z}} e_{n}$, where the energy density $e(t)=\left(e_{n}(t)\right)_{n \in \mathbb{Z}}$ is defined by

$$
e=\frac{\dot{x}^{2}}{2}+V\left(\delta^{+} x\right)
$$

We assume $\lim _{n \rightarrow \pm \infty} \dot{x}_{n}(t)=0, V(0)=0$ and $\lim _{n \rightarrow \pm \infty}\left(x_{n+1}(t)-x_{n}(t)\right)=0$. Using (5), it is lengthy but straightforward to check that

$$
\dot{e}+\delta^{-} q=-\left(\delta^{-} \dot{x}\right)^{2} W^{\prime \prime}\left(\delta^{-} x\right)
$$

where

$$
q=-V^{\prime}\left(\delta^{+} x\right)\left(1+\delta^{+}\right) \dot{x}-\dot{x} \frac{d}{d t} W^{\prime}\left(\delta^{+} x\right)
$$

Since $\lim _{n \rightarrow \pm \infty} q_{n}(t)=0$, equation (6) yields the dissipation inequality

$$
\dot{H}=-\left\|\left(\delta^{-} \dot{x}\right) \sqrt{W^{\prime \prime}\left(\delta^{-} x\right)}\right\|_{2}^{2} \leq 0,
$$

where \|\|$_{2}$ denotes the usual $\ell_{2}$ norm. In a similar manner, when considering infinite energy solutions (such as fronts), the dissipativity of the model is linked with the negativity of the right side of (6).

In what follows, we consider traveling waves propagating at constant velocity $c \neq 0$ :

$$
x_{n}(t)=X(\xi), \quad \xi=n-c t .
$$

We start by reformulating the above equations in the frame moving with the wave. Let us denote $e_{n}(t)=E(\xi),\left(\delta^{ \pm} X\right)(\xi)= \pm(X(\xi \pm 1)-X(\xi))$, and note that $\delta^{-}=\frac{d}{d \xi} \mathcal{A}$, where $\mathcal{A}$ is the averaging operator $(\mathcal{A} X)(\xi)=\int_{0}^{1} X(\xi-\varphi) d \varphi$. Substituting Ansatz (7) in (5) and integrating once with respect to $\xi$, we obtain the following nonlocal differential equation for the profile function $X$ :

$$
c^{2} X^{\prime}+\mathcal{A}\left(-V^{\prime}\left(\delta^{+} X\right)+c \frac{d}{d \xi} W^{\prime}\left(\delta^{+} X\right)\right)=C_{0},
$$

where $C_{0}$ is an arbitrary constant (independent of $\xi$ ). Similarly, using Ansatz (7) in (6) and dividing by $-c^{2}$ yields

$$
\frac{1}{c} \frac{d}{d \xi}(E+\mathcal{A} Q)=\left(\delta^{-} X^{\prime}\right)^{2} W^{\prime \prime}\left(\delta^{-} X\right) \geq 0
$$

where

$$
Q=-V^{\prime}\left(\delta^{+} X\right)\left(1+\delta^{+}\right) X^{\prime}+c X^{\prime} \frac{d}{d \xi} W^{\prime}\left(\delta^{+} X\right)
$$

Now let us concentrate on front solutions satisfying

$$
\lim _{\xi \rightarrow \pm \infty} X^{\prime}(\xi)=R_{ \pm \infty}
$$

with $R_{+\infty} \neq R_{-\infty}$. This implies

$$
\lim _{\xi \rightarrow \pm \infty}(X(\xi+1)-X(\xi))=R_{ \pm \infty}
$$

by the mean value equality. Consequently, $R_{ \pm \infty}=\lim _{n \rightarrow \pm \infty}\left(x_{n+1}(t)-x_{n}(t)\right)$ correspond to relative displacements at infinity. Particle velocities at infinity are given by

$$
\lim _{n \rightarrow \pm \infty} \dot{x}_{n}(t)=-c R_{ \pm \infty}
$$

Using the first integral (8) and letting $\xi \rightarrow \pm \infty$, one obtains the jump condition

$$
\llbracket R \rrbracket c^{2}=\llbracket V^{\prime} \rrbracket,
$$


where $\llbracket R \rrbracket=R_{+\infty}-R_{-\infty}, \llbracket V^{\prime} \rrbracket=V^{\prime}\left(R_{+\infty}\right)-V^{\prime}\left(R_{-\infty}\right)$. Consequently, a necessary condition for the existence of a front connecting $R_{ \pm \infty}$ is that $\llbracket R \rrbracket$ and $\llbracket V^{\prime} \rrbracket$ have the same sign.

Remark 1. The condition $\llbracket V^{\prime} \rrbracket \llbracket R \rrbracket>0$ is satisfied for strictly convex potentials $V$ and violated for strictly concave potentials, indicating in that case the nonexistence of traveling fronts.

Condition (13) implies

$$
c=\sigma\left(\frac{\llbracket V^{\prime} \rrbracket}{\llbracket R \rrbracket}\right)^{1 / 2}
$$

where $\sigma=\operatorname{sign}(c)= \pm 1$. This value of the wave velocity was previously derived in [3] (see also [23] for a derivation in a continuum limit). It is remarkable that the magnitude of the front velocity does not depend on the dissipation potential $W$.

Remark 2. In the conservative case $W^{\prime \prime}=0$, condition (13) was obtained in [21]. It can be also derived as a Rankine-Hugoniot jump condition from the p-system obtained in the hyperbolic limit of the lattice model (see e.g. [3, 20]).

In what follows, we assume $\llbracket V^{\prime} \rrbracket \llbracket R \rrbracket>0$ and determine the sign $\sigma$ using the dissipativity of the model. Let us denote by $\mathcal{I}$ the interval $\left(R_{\mp \infty}, R_{ \pm \infty}\right)$ and assume $W^{\prime \prime}>0$ almost everywhere on $\mathcal{I}$, hence the inequality in (9) becomes strict almost everywhere. Integrating (9) from $\xi=-\infty$ to $\xi=+\infty$ yields then

$$
\frac{1}{c}\left(\frac{c^{2}}{2}\left(R_{+\infty}^{2}-R_{-\infty}^{2}\right)+\llbracket V \rrbracket+V^{\prime}\left(R_{-\infty}\right) R_{-\infty}-V^{\prime}\left(R_{+\infty}\right) R_{+\infty}\right)>0 .
$$

Then the substitution of (13) in (15) leads to

$$
\frac{1}{c}\left(\llbracket V \rrbracket-\llbracket R \rrbracket \frac{V^{\prime}\left(R_{-\infty}\right)+V^{\prime}\left(R_{+\infty}\right)}{2}\right)>0,
$$

which allows us to determine the sign of $c$ :

$$
\sigma=\operatorname{sign}\left(\llbracket V \rrbracket-\llbracket R \rrbracket \frac{V^{\prime}\left(R_{-\infty}\right)+V^{\prime}\left(R_{+\infty}\right)}{2}\right) .
$$

The sign condition (16) was previously derived in [3] for a quadratic dissipation potential $W$.

From inequality (16), a necessary condition for the existence of a dissipative front connecting $R_{ \pm \infty}$ is that

$$
\llbracket V \rrbracket-\llbracket R \rrbracket \frac{V^{\prime}\left(R_{-\infty}\right)+V^{\prime}\left(R_{+\infty}\right)}{2} \neq 0 .
$$

The coefficient that appears in (18) can be interpreted geometrically [21]. Indeed, defining the rescaled force $f(r)=\llbracket R \rrbracket V^{\prime}(r)$ and the chord $T(s)=s f\left(R_{+\infty}\right)+(1-s) f\left(R_{-\infty}\right)$, we note that

$$
\llbracket V \rrbracket-\llbracket R \rrbracket \frac{V^{\prime}\left(R_{-\infty}\right)+V^{\prime}\left(R_{+\infty}\right)}{2}=\int_{0}^{1} f\left(s R_{+\infty}+(1-s) R_{-\infty}\right)-T(s) d s .
$$

Consequently, condition (18) indicates that the signed area between the graph of $f$ on $\mathcal{I}$ and the chord through $R_{ \pm \infty}$ does not vanish. Moreover, if $\llbracket R \rrbracket V^{(3)}<0$ on $\mathcal{I}$ then $f$ is strictly concave and $\sigma=+1$. On the contrary, if $\llbracket R \rrbracket V^{(3)}>0$ on $\mathcal{I}$ then $f$ is strictly convex and $\sigma=-1$.

Remark 3. If $V$ is quadratic on $\mathcal{I}$, there are no traveling front solutions because condition (18) is violated (since $f$ is an affine function, it coincides with the chord and the right side of (19) vanishes).

To our knowledge, the existence of dissipative front solutions under conditions (13)-(18) is an open problem. Let us assume that these conditions are satisfied with $V^{(3)} \neq 0$ on $\mathcal{I}$ and that a corresponding traveling front exists. From the above computations, we have $\sigma=-\operatorname{sign}\left(\llbracket R \rrbracket V^{(3)}\right)$. Consequently, if $V^{(3)}<0$ then $\sigma=\operatorname{sign}(\llbracket R \rrbracket)$ and thus the front propagates from the most compressed region towards 
the less compressed region (as already noticed in [3]), and the opposite occurs if $V^{(3)}>0$. In both cases, denoting $\llbracket \dot{x}_{n} \rrbracket=\lim _{n \rightarrow+\infty} \dot{x}_{n}(t)-\lim _{n \rightarrow-\infty} \dot{x}_{n}(t)$, we find $\llbracket \dot{x}_{n} \rrbracket=-c \llbracket R \rrbracket$ using (12), which leads to

$$
\llbracket \dot{x}_{n} \rrbracket=\left(\llbracket V^{\prime} \rrbracket \llbracket R \rrbracket\right)^{1 / 2} \operatorname{sign}\left(V^{(3)}\right) .
$$

In addition, let us assume $V$ convex and consider the strain-dependent sound velocity $\sqrt{V^{\prime \prime}}$. From the above remark on the direction of propagation, we conclude that the front propagates from the region of higher sound velocity towards the region of lower sound velocity, and the front velocity (14) lies between the two limiting sound velocities by the mean value theorem.

Remark 4. In the conservative case $W^{\prime \prime}=0$, the right side of equation (9) vanishes identically and condition (16) becomes

$$
\llbracket V \rrbracket-\llbracket R \rrbracket \frac{V^{\prime}\left(R_{-\infty}\right)+V^{\prime}\left(R_{+\infty}\right)}{2}=0 .
$$

Identity (20) corresponds to a necessary condition for the existence of a front connecting $R_{ \pm \infty}[3,21]$. In particular, if $V^{(3)}$ does not change sign on $\mathcal{I}$ then (20) is not satisfied (a consequence of identity (19)) and steady fronts do not exist. This applies in particular to the generalized Hertz potential defined in (4) with $\alpha>0$ and $\alpha \neq 1$. For existence results concerning traveling fronts in Fermi-Pasta-Ulam lattices (i.e. without dissipation) with appropriate interaction potentials, see [26, 21, 22].

To illustrate the above results, consider the generalized Hertz potential $V$ defined in (4) with $\alpha>0$ and $\alpha \neq 1$. We consider compression fronts with $R_{-\infty}=-\delta<0$ and $R_{+\infty}=0$ (i.e. the chain is at rest at $+\infty)$. We have $\llbracket R \rrbracket V^{(3)}(x)=\delta k \alpha(1-\alpha)|x|^{\alpha-2} H(-x)$, where $H$ denotes the Heaviside function. Consequently, $\sigma=\operatorname{sign}(\alpha-1)$ and

$$
c=\operatorname{sign}(\alpha-1) \sqrt{\frac{k}{m}} \delta^{(\alpha-1) / 2} .
$$

It follows that for $\alpha>1$, steady dissipative fronts propagate from the compressed region to the region where the chain is at rest, and the opposite occurs if $\alpha<1$. Particle velocities $v_{\mathrm{p}}$ at minus infinity are given by $v_{\mathrm{p}}=\lim _{n \rightarrow-\infty} \dot{x}_{n}(t)=c \delta$ according to (12), hence we obtain the following relations between $v_{\mathrm{p}}, \delta$ and $c$ :

$$
\begin{gathered}
v_{\mathrm{p}}=\operatorname{sign}(\alpha-1) \sqrt{\frac{k}{m}} \delta^{(\alpha+1) / 2}, \\
c=\operatorname{sign}(\alpha-1)\left(\frac{k}{m}\right)^{1 /(\alpha+1)}\left|v_{\mathrm{p}}\right|^{(\alpha-1) /(\alpha+1)} .
\end{gathered}
$$

This result agrees with the value obtained in $[19,39]$ using a formal continuum limit (see also $[40,15]$ ).

\section{DYNAMICAL PROPERTIES OF DISSIPATIVE GRANULAR CHAINS}

In this section, we describe some properties of dissipative granular chains endowed with the viscoelastic contact models (2). Section 3.1 recalls some scaling properties of the models and their consequences for the energy loss during contacts. These results have been previously obtained in [37] using a slightly different method, and in [13] for Hertzian elastic interactions (case $\alpha=3 / 2$ in (2)). We also apply the scaling properties to the normalization of front solutions. Section 3.2 recalls dynamical variables introduced in [30] for the numerical simulation of the models, which are adapted to the limited smoothness of the viscoelastic contact force. In section 3.3, we simulate the KK model (case $\alpha=\beta=3 / 2$ ) with a constant pressure applied at one end of the chain, and show that underdamped or overdamped fronts are generated depending on parameter values. 
3.1. Models and scaling properties. We consider a dissipative granular chain consisting of $N$ identical elements and described by the model (1)-(2). The dynamical equations read

$$
\begin{aligned}
m \ddot{x}_{n}= & k\left(x_{n-1}-x_{n}\right)_{+}^{\alpha}+\beta \gamma_{0} k\left(x_{n-1}-x_{n}\right)_{+}^{\beta-1}\left(\dot{x}_{n-1}-\dot{x}_{n}\right) \\
& -k\left(x_{n}-x_{n+1}\right)_{+}^{\alpha}-\beta \gamma_{0} k\left(x_{n}-x_{n+1}\right)_{+}^{\beta-1}\left(\dot{x}_{n}-\dot{x}_{n+1}\right), \quad 1 \leq n \leq N .
\end{aligned}
$$

We shall consider different types of boundary conditions. Firstly, Neumann boundary conditions

$$
x_{0}=x_{1}+\delta, \quad x_{N+1}=x_{N}-\tilde{\delta},
$$

correspond to fixing a constant pressure at each end of the chain, the cases $\tilde{\delta}=0$ or $\delta=0$ corresponding to free end boundary conditions. We shall also consider periodic boundary conditions

$$
x_{0}=x_{N}-L, \quad x_{N+1}=x_{1}+L, \quad \dot{x}_{0}=\dot{x}_{N}, \quad \dot{x}_{N+1}=\dot{x}_{1},
$$

where $x_{n}$ can be interpreted as a rescaled angular coordinate. In that case, the relative displacements $u_{n}=x_{n}-x_{n+1}$ satisfy $u_{0}=u_{N}$ and we have

$$
\sum_{n=0}^{N-1} u_{n}=x_{0}-x_{N}=-L=x_{1}-x_{N+1}=\sum_{n=1}^{N} u_{n} .
$$

The model (24) can be suitably renormalized given a reference velocity $v_{0}>0$, which typically corresponds to the velocity of an impacting bead or a piston. This leads us to introduce the time and length scales

$$
T_{0}=\left(\frac{m}{k}\right)^{1 /(\alpha+1)} v_{0}^{(1-\alpha) /(\alpha+1)}, \quad \delta_{0}=v_{0} T_{0}=\left(\frac{m}{k}\right)^{1 /(\alpha+1)} v_{0}^{2 /(\alpha+1)} .
$$

These scales indicate how collision time and maximal indentation scale with parameters for a two-bead elastic collision [37]. Setting $x_{n}(t)=\delta_{0} \tilde{x}_{n}(\tilde{t})$ with $\tilde{t}=t / T$, and dropping the tilde for convenience, equation (24) becomes

$$
\begin{aligned}
\ddot{x}_{n}= & \left(x_{n-1}-x_{n}\right)_{+}^{\alpha}+\beta \gamma\left(x_{n-1}-x_{n}\right)_{+}^{\beta-1}\left(\dot{x}_{n-1}-\dot{x}_{n}\right) \\
& -\left(x_{n}-x_{n+1}\right)_{+}^{\alpha}-\beta \gamma\left(x_{n}-x_{n+1}\right)_{+}^{\beta-1}\left(\dot{x}_{n}-\dot{x}_{n+1}\right), \quad 1 \leq n \leq N,
\end{aligned}
$$

with the dimensionless damping parameter

$$
\gamma=\gamma_{0} v_{0}^{\frac{2 \beta}{\alpha+1}-1}\left(\frac{k}{m}\right)^{1-\frac{\beta}{\alpha+1}} .
$$

Remark 5. Alternatively, if $\delta>0$ in (25) so that a constant pressure is applied at one end of the chain, one can set the length scale to $\delta_{0}=\delta$ and determine the velocity scale $v_{0}$ and time scale $T_{0}$ with (27), which leads to the rescaled equation (28).

The weak dissipation limit corresponds to $\gamma \approx 0$ in (28). Interestingly, this case occurs either for small or large enough impact velocities depending on the exponents $\alpha, \beta$. More precisely, let $v_{\mathrm{c}}\left(\gamma_{0}, k, m\right)$ denote the value of $v_{0}$ such that $\gamma=1$ in (29). When $\beta>(\alpha+1) / 2$, weak dissipation occurs for $v_{0} \ll v_{\mathrm{c}}$. This is the case in particular for $\alpha>1$ and $\beta \geq \alpha$, as in the generalized KK model $(\beta=\alpha)$ and the Simon-Hunt-Crossley model with $\beta=\alpha+1[50,25]$. On the contrary, when $\beta<(\alpha+1) / 2$, weak viscoelastic dissipation occurs for $v_{0} \gg v_{\mathrm{c}}$. This case occurs for example if $\alpha>1$ and $\beta=1$ (i.e. each contact consists of a nonlinear spring with linear dashpot), a class of models studied in $[19,36]$ in the context of shock waves. Moreover, in the degenerate case $\beta=(\alpha+1) / 2$, the dimensionless damping parameter $\gamma$ becomes independent of the reference velocity $v_{0}$.

Remark 6. A way to estimate $\gamma$ is through the coefficient of restitution e, defined as minus the ratio of the relative velocities of two beads before and after an impact. For example, consider two identical impacting beads of mass $m$, with a contact law corresponding to the KK model $(\beta=\alpha=3 / 2$, Hertzian stiffness $k$, dissipation constant $\gamma_{0}$ ). When $e \approx 1$ (or equivalently $\gamma \approx 0$ ) one has $\gamma \approx 0.438(1-e)$ 
(see e.g. [45]). For example, from restitution coefficients obtained experimentally in [35] with different types of beads and for an impact velocity $v_{0}=0.5 \mathrm{~m} \mathrm{~s}^{-1}$, one can infer that $\gamma \approx 0.008$ for glass spheres with radius $R=1.96 \mathrm{~cm}, \gamma \approx 0.05$ for steel spheres with $R=1.65 \mathrm{~cm}$, and $\gamma \approx 0.13$ for cork spheres with a lead core $(R=1.66 \mathrm{~cm}, m=31.7 \mathrm{~g})$.

We end this section by connecting the results of section 2 to the above scaling properties. Let us consider model (24) for $\alpha>1$ in the limit when the number $N$ of particles tends towards infinity. Assume the existence of a traveling front connecting the compressed state $x_{n}-x_{n+1}=\delta>0, \dot{x}_{n}=v_{\mathrm{p}}$ when $n \rightarrow-\infty$ to the chain at rest when $n \rightarrow+\infty$. Choosing the velocity scale $v_{0}=v_{\mathrm{p}}$, one obtains $\delta_{0}=\delta$ by (22) and (27). Consequently, the rescaled front solution of (28) satisfies $\lim _{n \rightarrow-\infty} \dot{x}_{n}=1$ and $\lim _{n \rightarrow-\infty}\left(x_{n}-x_{n+1}\right)=1$, and thus the rescaled front velocity $c$ is equal to unity thanks to (21). Moreover, once $\alpha$ and $\beta$ are fixed, the influence of the other parameters on the qualitative properties of the front (shape, stability) can be examined by resorting to the dimensionless parameter $\gamma$ defined in (29). In particular, for $\beta=\alpha$, this parameter takes the form

$$
\gamma=\gamma_{0} v_{\mathrm{p}}^{\frac{\alpha-1}{\alpha+1}}\left(\frac{k}{m}\right)^{\frac{1}{\alpha+1}}=\gamma_{0} \delta^{(\alpha-1) / 2} \sqrt{\frac{k}{m}}=\gamma_{0} c .
$$

3.2. Choice of dynamical variables. Let us consider equation (28) for $\alpha>1$ and $\beta \in[1,2)$. In this range of parameters, the model presents a lack of smoothness because the map defining the right-hand side is discontinuous accross the hyperplanes $x_{n-1}=x_{n}$ for $\beta=1$, and not Lipschitz continuous for $\beta \in(1,2)$, in particular for the KK model. This lack of regularity can induce a rather severe decrease of the order of convergence of some classical numerical time-integration schemes, as shown in [30], and may as well deteriorate the computation of wave profiles based on Newton-type methods. However, these difficulties can be circumvented to some extent by an appropriate choice of dynamical variables [30]. Defining a generalized velocity $w_{n}$ through

$$
\dot{x}_{n}=w_{n}+\gamma\left(\left(x_{n-1}-x_{n}\right)_{+}^{\beta}-\left(x_{n}-x_{n+1}\right)_{+}^{\beta}\right),
$$

the dynamical equation (24) becomes

$$
\dot{w}_{n}=\left(x_{n-1}-x_{n}\right)_{+}^{\alpha}-\left(x_{n}-x_{n+1}\right)_{+}^{\alpha} .
$$

Let us denote $x=\left(x_{1}, \ldots, x_{N}\right), w=\left(w_{1}, \ldots, w_{N}\right)$ and consider $Y=(x, w)$ as a new dynamical variable. For all $\beta \geq 1$, the right-hand side of (31)-(32) defines a locally Lipschitz continuous map $Y \mapsto F_{\mathrm{S}}(Y)\left(F_{\mathrm{S}}\right.$ is even $C^{1}$ for $\left.\beta>1\right)$ and thus the corresponding initial value problem is well posed according to the Cauchy-Lipschitz theorem. Moreover, as shown in [30] for several numerical timeintegration schemes, the improved regularity of the right-hand side brings better convergence properties compared to the differential equation based on the usual dynamical variables $\left(x_{n}, \dot{x}_{n}\right)$.

Remark 7. The case $\alpha \in(0,1)$ has been also investigated in recent studies, see [54] and references therein. However, to our knowledge both the well-posedness of the initial value problem (uniqueness of solutions) in the presence of gap openings and the properties of classical numerical time-integration methods (convergence, stability) have not been analyzed yet.

The above framework extends to an infinite number of particles, with the boundary condition (25) replaced by

$$
\lim _{n \rightarrow-\infty}\left(x_{n}(t)-x_{n+1}(t)\right)=\delta, \quad \lim _{n \rightarrow+\infty}\left(x_{n}(t)-x_{n+1}(t)\right)=\tilde{\delta} .
$$

A possible choice of functional setting can be made as follows. Let us fix $\tilde{\delta}=0$ for simplicity and consider the piecewise-linear sequence $\bar{x}_{n}=\max (-n \delta, 0)$ which satisfies (33). We consider the Banach space

$$
\mathcal{Y}=\left\{y=\left(y_{n}\right)_{n \in \mathbb{Z}} \subset \mathbb{R}, \lim _{n \rightarrow-\infty} y_{n} \text { exist in } \mathbb{R}, \lim _{n \rightarrow+\infty} y_{n}=0\right\}
$$


endowed with the supremum norm \|\|$_{\infty}$, and the associated affine metric space

$$
\mathcal{X}=\left\{x=\left(x_{n}\right)_{n \in \mathbb{Z}}, x-\bar{x} \in \mathcal{Y}\right\} .
$$

System (31)-(32) is considered for $Y(t)=(x(t), w(t)) \in \mathcal{X} \times \mathcal{Y}$. The right-hand side $F_{\mathrm{s}}: \mathcal{X} \times \mathcal{Y} \rightarrow$ $\mathcal{Y} \times \mathcal{Y}$ is $C^{1}$ for $\beta>1$, and thus the initial value problem is locally well posed in $\mathcal{X} \times \mathcal{Y}$. Moreover, $v=\lim _{n \rightarrow-\infty} w_{n}$ is a conserved quantity $\left(\dot{v}=\lim _{n \rightarrow-\infty} \dot{w}_{n}=0\right.$ using (32)) and (31) leads to

$$
\lim _{n \rightarrow-\infty} \dot{x}_{n}(t)=v
$$

3.3. Initiation of fronts in dissipative granular chains described by the KK model. In this section we illustrate the initiation of fronts using numerical computations. We use formulation (31)-(32) for time-integration, and restrict our study to the KK model with standard parameter values $\beta=\alpha=3 / 2$. Computations are performed for a chain of $N=420$ particles initially at rest. Boundary conditions take the form (25), with a free end boundary condition at $x_{N}$ (i.e. $\tilde{\delta}=0$ ) and a constant compression $\delta$ at the first element for $t \geq 0$. Time-integration is performed using the standard ODE solver of the software package Scilab (www. scilab.org), with relative and absolute tolerances set to $10^{-13}$ and $10^{-15}$ respectively.

The response of the chain is described in figures 1,2 and 3 for $\delta=0.1$. After a transient, a front is established with velocity $c=\delta^{(\alpha-1) / 2} \approx 0.56$ determined by (21). This value does not depend on $\gamma$, but dissipation strongly affects the front shape. When $\gamma$ is small enough, oscillations appear behind the shock, as shown in figure 1 (underdamped regime). These oscillations can induce a rather large overstrike, i.e. a significant increase of precompression compared to $\delta$ and of bead velocities compared to $v_{\mathrm{p}}=\delta c$ (in the simulation of figure 1 , the maximal elastic force induced by the traveling front is roughly 1.6 times the applied pressure). If $\gamma$ is large enough, the asymptotic oscillations around the compressed state disappear (overdamped regime) and the overstrike becomes very small (the fronts look almost monotonic), see the left plot in figure 3. This transition occurs above a critical damping $\gamma_{c}$ proportional to $\delta^{(1-\alpha) / 2}$ or $v_{\mathrm{p}}^{(1-\alpha) /(1+\alpha)}$ equivalently, according to the scaling properties described in section 3.1 (the normalization of front solutions yields the rescaled damping (30)).

Figure 2 illustrates the propagation and multiple reflections of the front solution excited in the simulation of figure 1 for $\gamma=0.06$. One can notice the more diffusive (thickening) domain wall of the backward propagating fronts, indicating that they do not correspond to traveling waves, in agreement with the results of section 2 . The velocities remain positive and tend to increase with time. Indeed, the dynamical equations (28) yield $\frac{d}{d t} \sum_{n=1}^{N} \dot{x}_{n}=\delta^{\alpha}$ due to the constant pressure at one end, therefore the average velocity in the chain is $\delta^{\alpha} t / N$. As shown in the right plot in figure 3 for $\gamma=0.65$, the same phenomena occur in the overdamped regime.

In figure 2 for $\gamma=0.06$, one can also notice that gap openings occur, as some beads are ejected when the front initially reaches the end of the chain at $t \approx 750$. For $\gamma=0.65$ (overdamped regime), gap openings do not occur, as shown in the right plot of figure 3. In both case, the renormalized chain compression (or mean relative displacement) $\frac{1}{N}\left(x_{1}(t)-x_{N}(t)\right)=\frac{1}{N} \sum_{n=1}^{N-1} u_{n}(t)$ oscillates around a mean value relatively close to $\delta / 2$ as the shocks propagate back and forth.

In the next sections, we will introduce amplitude equations allowing to approximate the front shape. Depending on the overdamped or underdamped character of the front, and size of the overstrike, it will be more or less important to take into account dispersive effects. This will lead us to the logarithmic Burgers equation if dispersion is neglected (section 4) and to the logarithmic KdV Burgers equation if dispersion is taken into account (section 5). 

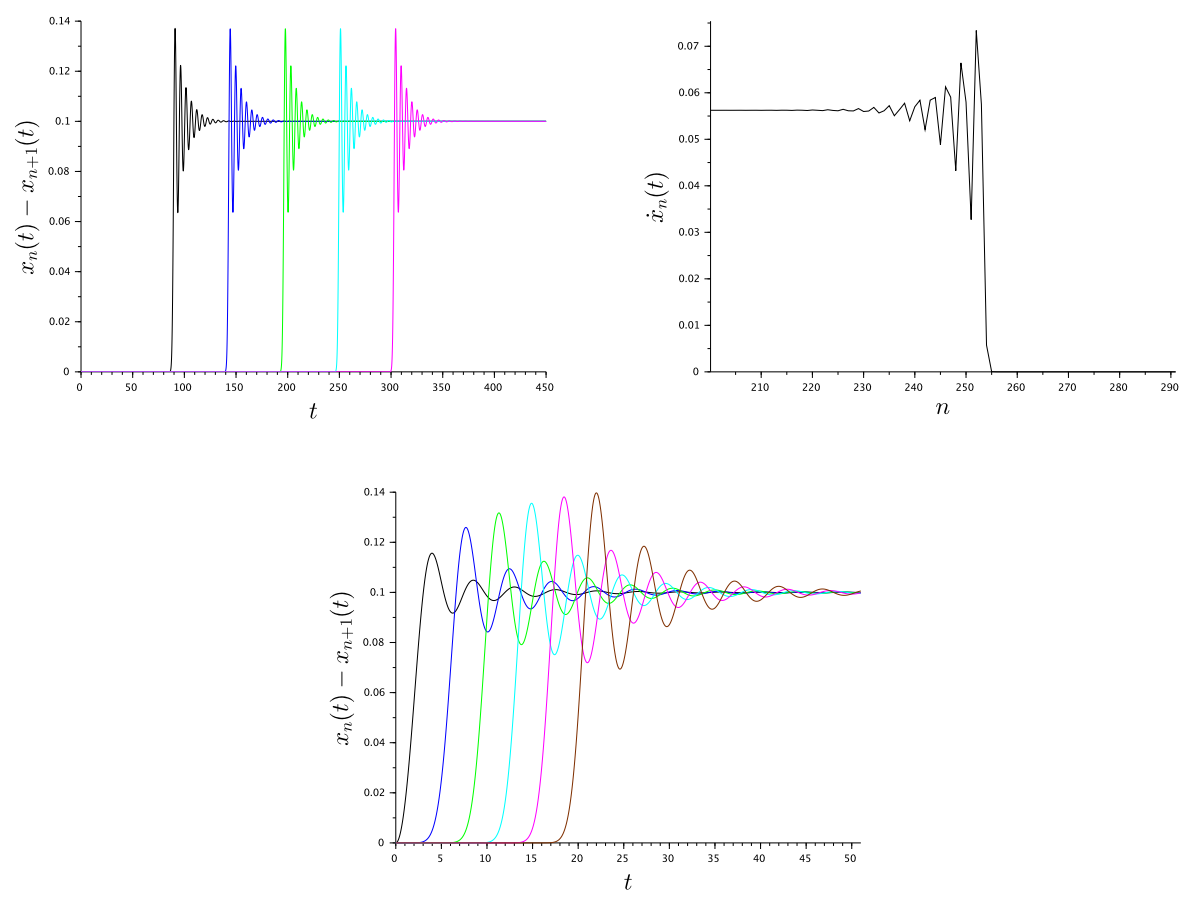

FIGURE 1. Front excited by applying a constant pressure (with $\delta=0.1$ ) at one end of the chain, for $\alpha=3 / 2$ and $\gamma=0.06$. The top plots describe the dynamics when a steady regime is reached. In the top left plot, relative displacements are plotted versus time for $n=50$ (black), $n=80$ (blue), $n=110$ (green), $n=140$ (light blue), and $n=170$ (magenta). In the top right plot, velocities are plotted versus $n$ at $t=450$ (the front propagates rightwise). The bottom plot describes the transient regime where the amplitude of the wave varies as it propagates through the first elements of the chain. Relative displacements are plotted versus time for odd values of $n$ between $n=1$ and $n=11$, from left to right (black: $n=1$, blue: $n=3$, green: $n=5$, light blue: $n=7$, magenta: $n=9$, brown: $n=11$ ).

\section{Logarithmic Burgers limit of The KuwABARA-Kono MOdel}

4.1. Derivation of the logarithmic Burgers equation. The generalized KK model corresponds to the case $\beta=\alpha$ of (28) :

$$
\ddot{x}=-\delta^{-}\left(1+\gamma \frac{d}{d t}\right)\left(-\delta^{+} x\right)_{+}^{\alpha},
$$

where $\gamma>0$ is the nondimensional dissipation constant. We consider an infinite chain with $x(t)=$ $\left(x_{n}(t)\right)_{n \in \mathbb{Z}}$ and introduce the variable $u=-\delta^{+} x$ corresponding to relative displacements. Its evolution is described by the equation

$$
\ddot{u}=\left(1+\gamma \frac{d}{d t}\right) \Delta_{\mathrm{d}} u_{+}^{\alpha},
$$

where $\Delta_{\mathrm{d}}=\delta^{+} \delta^{-}=\delta^{+}-\delta^{-}$is the usual discrete Laplacian.

We assume $u \geq 0$ (absence of gaps between beads) and consider the Ansatz

$$
u_{n}(t)=\delta y(\xi, \tau)^{1 / \alpha},
$$

where the new variable $y$ can be interpreted as a rescaled force variable. The moving frame coordinate $\xi=\epsilon(n-c t)$ with $c=\delta^{(\alpha-1) / 2}$ accounts for the propagation of disturbances at the velocity determined 

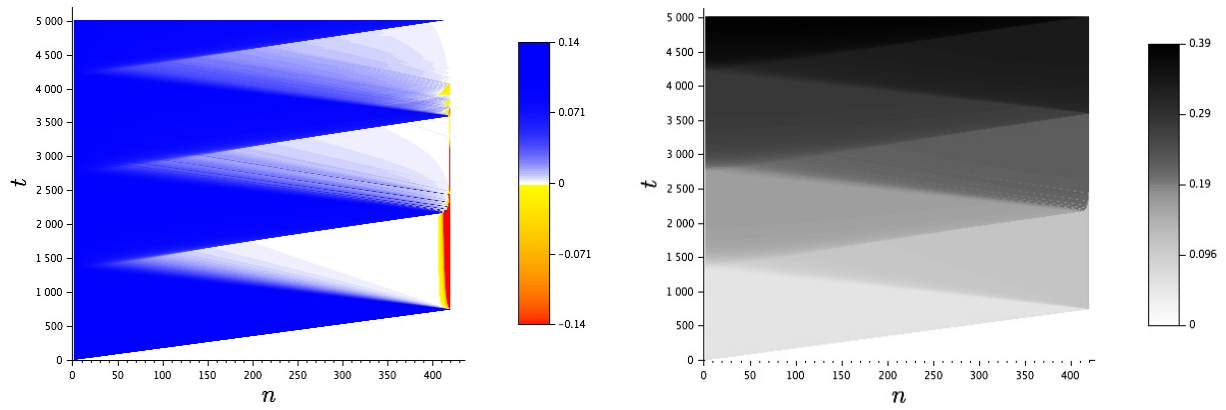

FIGURE 2. Space-time diagrams showing relative displacements $x_{n}(t)-x_{n+1}(t)$ (left plot) and velocities $\dot{x}_{n}(t)$ (right plot) for the numerical simulations of figure 1 for $\gamma=0.06$. In the left plot, the red color corresponds to values less that -0.14 . The minimum relative displacements are around -9.83 at $t \approx 2326$, when the second forward-propagating shock reaches the end of the chain and new beads start to be ejected.
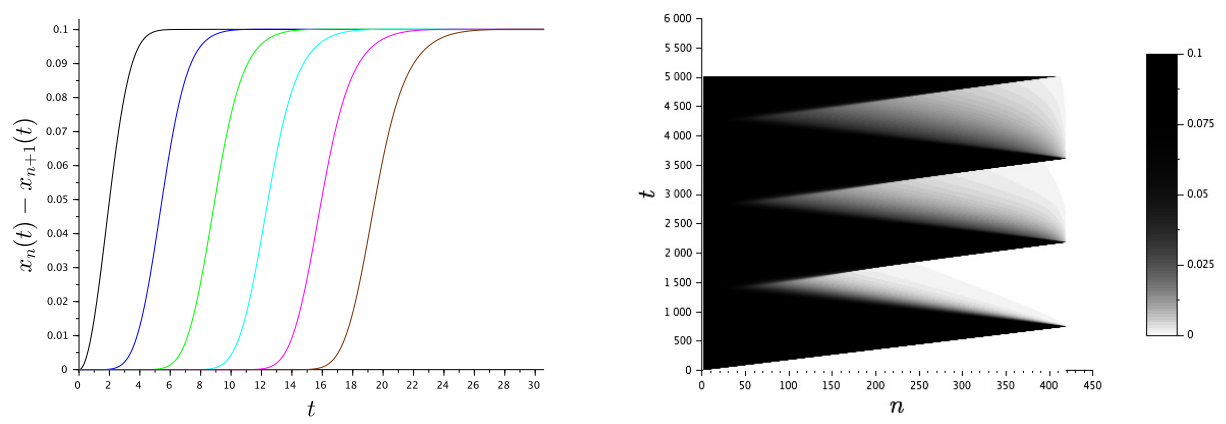

FIGURE 3. Front excited by applying a constant pressure (with $\delta=0.1$ ) at one end of the chain, for $\alpha=3 / 2$ and $\gamma=0.65$. The left plot describe the transient regime where the amplitude of the wave varies slightly as it propagates through the first elements of the chain. Relative displacements are plotted versus time for odd values of $n$ between $n=1$ and $n=11$, from left to right (black: $n=1$, blue: $n=3$, green: $n=5$, light blue: $n=7$, magenta: $n=9$, brown: $n=11$ ). The steady front which is formed asymptotically does not display oscillations in that case. The right plot shows a spacetime diagram of relative displacements on a longer time scale. This reveals the steady front propagation and multiple reflections.

by (21). The slow time variable $\tau=\frac{\gamma}{2} \epsilon^{2} c^{2} t$ captures the time modulation induced by diffusive effects originating from the contact damping. In the following computation, we shall consider $\alpha-1$ as a small parameter related to $\epsilon$, in such a way that nonlinear effects act on the same time scale as diffusive terms.

From the chain rule and a Taylor expansion, we note that

$$
\frac{d}{d t}=c\left(\frac{\gamma}{2} \epsilon^{2} c \partial_{\tau}-\epsilon \partial_{\xi}\right), \quad \Delta_{\mathrm{d}}=\epsilon^{2} \partial_{\xi}^{2}+O\left(\epsilon^{4}\right) \text { when } \epsilon \rightarrow 0
$$

Consequently, assuming $\epsilon \ll \min (1, \gamma c)$, one can neglect the $O\left(\epsilon^{4}\right)$ terms in equation (36) (in particular dispersive effects) and keep the $O\left(\epsilon^{3} \gamma c\right)$ terms, which yields

$$
\left(\partial_{\xi}^{2}-\gamma c \epsilon \partial_{\xi \tau}^{2}\right) y^{1 / \alpha}=\partial_{\xi}^{2}\left(1-\gamma c \epsilon \partial_{\xi}\right) y .
$$


Integrating in $\xi$ and rearranging terms, we get

$$
\partial_{\tau}\left(y^{1 / \alpha}\right)+\frac{1}{\gamma c \epsilon} \partial_{\xi}\left(y-y^{1 / \alpha}\right)=\partial_{\xi}^{2} y .
$$

This leads us to set $\epsilon=\frac{\alpha-1}{\alpha \gamma c}$, so that $\gamma c \epsilon=1-\frac{1}{\alpha}$ and equation (39) reads

$$
\partial_{\tau}\left(y^{1 / \alpha}\right)+\partial_{\xi} f_{\alpha}(y)=\partial_{\xi}^{2} y
$$

with $f_{\alpha}(y)=\left(y-y^{1 / \alpha}\right) /\left(1-\frac{1}{\alpha}\right)$. Then letting $\alpha \rightarrow 1^{+}$in (40) yields the limiting problem

$$
\partial_{\tau} y+\partial_{\xi}(y \ln y)=\partial_{\xi}^{2} y
$$

i.e. a logarithmic Burgers equation [47]. Note that to be consistent with the assumption $\epsilon \ll \min (1, \gamma c)$, we must have $\left(1-\frac{1}{\alpha}\right)^{1 / 2} \ll \gamma c$.

In order to recover bead velocities from $y$, we come back to equation (35) which can be rewritten

$$
\frac{d \dot{x}}{d t}=-\delta c^{2}\left(1+\gamma \frac{d}{d t}\right) \delta^{-} y
$$

Using a Taylor expansion, we have

$$
-c \delta^{-}=-c\left(\epsilon \partial_{\xi}-\frac{\epsilon^{2}}{2} \partial_{\xi}^{2}+O\left(\epsilon^{3}\right)\right)=\frac{d}{d t}\left(1-\frac{\epsilon}{2} \partial_{\xi}\right)+O\left(\epsilon^{2}\right) .
$$

This allows us to integrate both sides of (42) with respect to time, leading to

$$
\dot{x}=\delta c\left(1-\gamma c \epsilon \partial_{\xi}\right)\left(1-\frac{\epsilon}{2} \partial_{\xi}\right) y+O\left(\epsilon^{2}\right),
$$

where an integration constant has been set to 0 by assuming the chain at rest at $+\infty$. Neglecting $O\left(\epsilon^{2}\right)$ terms yields finally to the approximation

$$
\dot{x} \approx \delta^{(\alpha+1) / 2}\left(y-\epsilon\left(\gamma c+\frac{1}{2}\right) \partial_{\xi} y\right) .
$$

In the particular case of stationary solutions, integrating (40) once with respect to $\xi$ allows one to express $y^{\prime}(\xi)$ as function of $y$. Back substitution in (43) yields the approximation

$$
\dot{x} \approx \delta^{(\alpha+1) / 2}\left(y^{1 / \alpha}-\frac{1}{2 \gamma c}\left(y-y^{1 / \alpha}\right)\right) .
$$

It is interesting to visualize the approximation of nonlinear terms realized by passing from equation (40) to (41), see the left panel of figure 4. For $\alpha$ rather close to unity (e.g. $\alpha=1.1$ ), the approximation is quite precise (compare the green and black graphs). For the classical value $\alpha=3 / 2$, the approximation is rather crude but remains meaningful, especially for $y>0.5$ (blue and black graphs). Note that one could derive in a similar manner a logarithmic Burgers equation for the rescaled relative displacements $y^{1 / \alpha}$, but for stationary solutions the formulation based on the force variable has the advantage of approximating only the single nonlinear term $f_{\alpha}$ instead of two $\alpha$-dependent terms.

4.2. Front solutions. Equation (41) admits a stationary front solution $y_{1}$ satisfying $\lim _{\xi \rightarrow-\infty} y_{1}(\xi)=$ 1 and $\lim _{\xi \rightarrow+\infty} y_{1}(\xi)=0$ with superexponential decay at $+\infty$ [47]. This solution satisfies $y^{\prime}=y \ln y$ and reads

$$
y_{1}(\xi)=2^{-\alpha e^{\xi}}
$$

where we have fixed the phase according to $y_{1}(0)=(1 / 2)^{\alpha}$, so that the rescaled relative displacements $u(\xi)=y_{1}(\xi)^{1 / \alpha}$ satisfy $u(0)=1 / 2$.

Equation (40) admits a semicompact stationary front solution $y_{\alpha}$ similar to the ones considered in [47], which satisfies $y^{\prime}=f_{\alpha}(y)$. With the same phase condition as above, this solution reads

$$
y_{\alpha}(\xi)=\left(1-\left(1-2^{1-\alpha}\right) e^{\xi}\right)^{\alpha /(\alpha-1)} \quad \text { for } \xi \leq \xi_{0}
$$



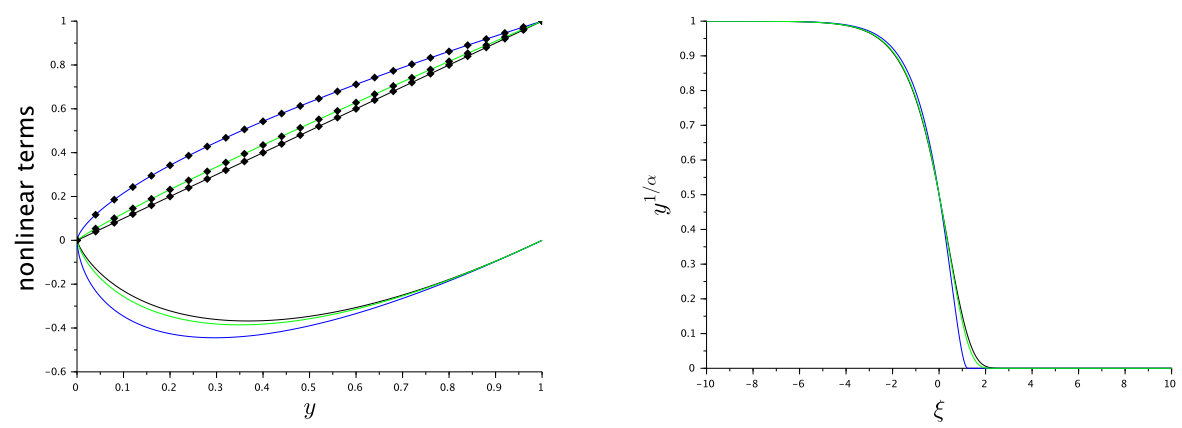

FIGURE 4. Left : nonlinear terms $f_{\alpha}(y)$ (full lines) and $y^{1 / \alpha}$ (dotted lines) of equation (40), for $\alpha=3 / 2$ (blue), $\alpha=1.1$ (green) and $\alpha \rightarrow 1^{+}$(black). Right : profiles of rescaled relative displacements $y^{1 / \alpha}$ corresponding to the stationary front solutions $y_{\alpha}$ of equation (40) for $\alpha=3 / 2$ (blue), $\alpha=1.1$ (green) and equation (41) corresponding to $\alpha \rightarrow 1^{+}$(black).

with $\xi_{0}=-\ln \left(1-2^{1-\alpha}\right)$ and $y_{\alpha}(\xi)=0$ for $\xi \geq \xi_{0}$. One can readily check that $y_{\alpha}(\xi) \sim y_{1}(\xi)$ when $\xi$ is fixed and $\alpha \rightarrow 1^{+}$. Moreover, as shown in the right panel of figure 4 , the rescaled relative displacements $y_{\alpha}^{1 / \alpha}$ and $y_{1}^{1 / \alpha}$ are close for $\alpha \in(1,3 / 2]$, so we shall resort to the analytical solution (45) which has a simpler form.

Using (45) yields the approximate relative displacements

$$
u_{n}(t)=\delta u(\xi),
$$

with $u(\xi)=y_{1}(\xi)^{1 / \alpha}=2^{-e^{\xi}}, \xi=\frac{\alpha-1}{\alpha \gamma c}(n-c t), c=\delta^{(\alpha-1) / 2}$. Moreover, we deduce from (44) the approximate velocities

$$
\dot{x}_{n}(t) \approx \delta^{(\alpha+1) / 2}\left(u-\frac{1}{2 \gamma c}\left(u^{\alpha}-u\right)\right)(\xi) .
$$

4.3. Dynamical simulations. In what follows, we compare the dynamical evolution of a localized perturbation for model (41) and the generalized KK model. The comparison of traveling front solutions will be performed in section 6.3. In the following dynamical simulations, we fix $\gamma=0.24$ and consider two values of the exponent $\alpha=1.1$ and $\alpha=3 / 2$.

We consider the initial condition $y(\xi, 0)=y^{(0)}(\xi)$ in (41) defined by

$$
y^{(0)}(\xi)=\left\{\begin{array}{c}
1 \text { for }|\xi| \leq 10 \\
\max \left(1-(|\xi|-10)^{2}, \eta\right) \text { for } 10 \leq|\xi| \leq \ell
\end{array}\right.
$$

with $\eta=10^{-7}$. The small parameter $\eta$ is introduced to avoid the singularity of the logarithmic nonlinearity in (41). We consider periodic boundary conditions $y(\xi+2 \ell, \tau)=y(\xi, \tau)$ with $\ell=\epsilon(p-1 / 2)$ for some integer $p$, where we fix $p=80$. The small parameter that determines the slow spatial and temporal scales in the chain of beads is $\epsilon=\frac{\alpha-1}{\alpha \gamma} \delta^{(1-\alpha) / 2}$ and we fix $\delta=5$.

Similarly, the generalized KK model is equiped with periodic boundary conditions (26) with $N=$ $2 p-1$ elements. We consider the initial condition defined by $u_{n}(0)=\delta\left(y^{(0)}(\epsilon(n-p))\right)^{1 / \alpha}$,

$$
\begin{gathered}
x_{N}(0)=0, \quad \forall n=1, \ldots, N-1: x_{n}(0)=\sum_{k=n}^{N-1} u_{k}(0), \\
\dot{x}_{n}(0)=\delta^{(\alpha+1) / 2} y^{(0)}(\epsilon(n-p))
\end{gathered}
$$


and $L=-\sum_{n=1}^{N} u_{n}(0)$ in (26). The velocity profile corresponds to the 0 th order approximation in (43). The initial condition is shown in figure 5. The generalized KK model is integrated using the formulation (31)-(32) as in section 3.3.
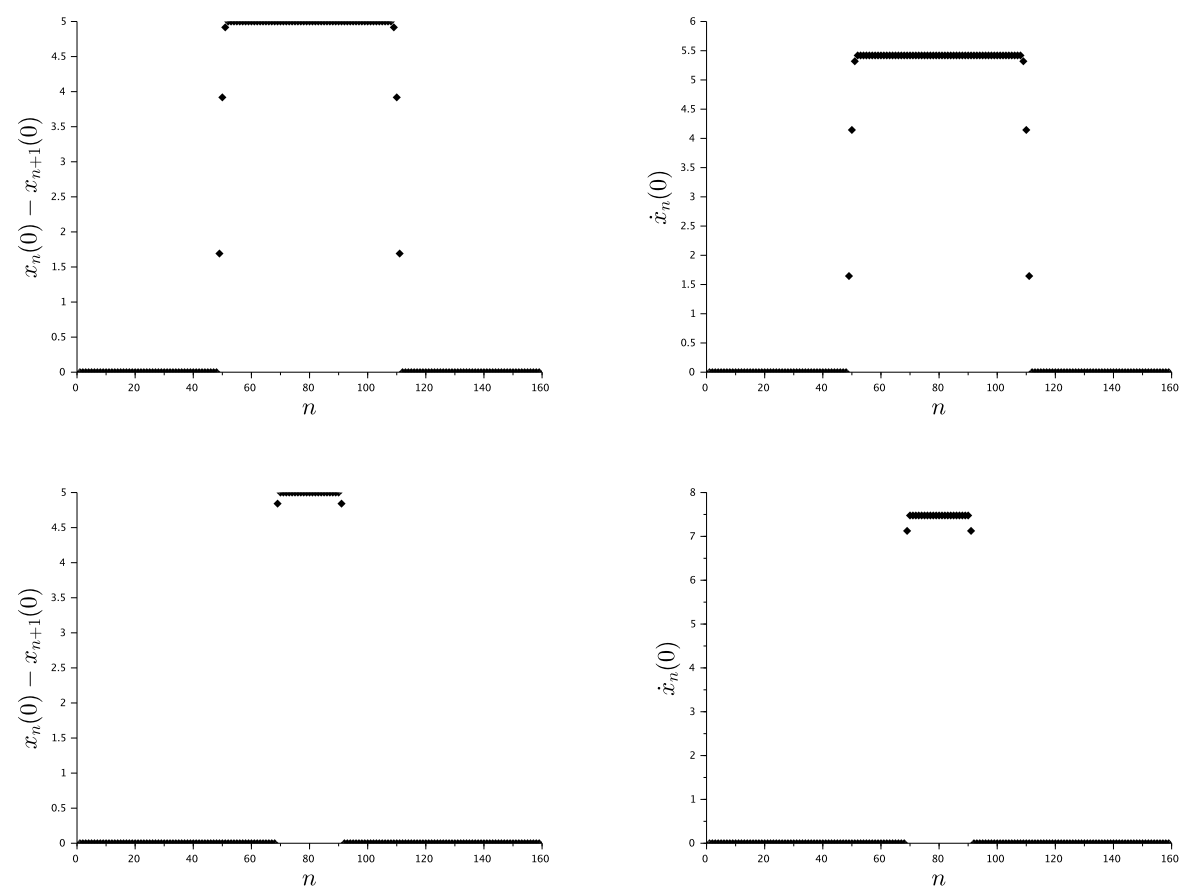

FIGURE 5. Initial condition considered for the comparison of the logarithmic Burgers equation to the original lattice model. Left : relative displacements, and right : velocities. Top : $\alpha=1.1$, and bottom : $\alpha=3 / 2$.

To discretize equation (41), we first set $y(\xi, \tau)=Y(\tilde{\xi}, \tau)$ with $\tilde{\xi}=\xi-\tau$. This maps (41) to the equation

$$
\partial_{\tau} Y+\ln Y \partial_{\tilde{\xi}} Y=\partial_{\tilde{\xi}}^{2} Y
$$

with advection velocity $\ln Y \leq 0$ for $Y \in(0,1]$ and the initial condition $Y_{\tau=0}=y^{(0)}$. Equation (50) is discretized using the explicit upwind finite difference scheme [1] with the space step $d \tilde{\xi}=2 \ell \times 10^{-4}$. The time step is fixed according to $d \tau=\frac{4}{5} d \tau_{\max }$ with $d \tau_{\max }=\frac{d \tilde{\xi}^{2}}{2+c_{\max } d \tilde{\xi}}$ and $c_{\max }=|\ln \eta|$ (Courant, Friedrichs, Lewy condition [1]).

According to formulas (37) and (43) (keeping only the leading order term), the approximate relative displacements and velocities resulting from the logarithmic Burgers approximation read

$$
u_{n}(t)=\delta Y(\tilde{\xi}, \tau)^{1 / \alpha}, \quad \dot{x}_{n}(t)=\delta^{(\alpha+1) / 2} Y(\tilde{\xi}, \tau),
$$

with $\tilde{\xi}=\epsilon(n-\tilde{c} t), \tilde{c}=\delta^{(\alpha-1) / 2}+\frac{\gamma}{2} \epsilon \delta^{\alpha-1}$ and $\tau=\frac{\gamma}{2} \epsilon^{2} \delta^{\alpha-1}$.

Figure 6 compares the approximate solution obtained by integrating the logarithmic Burgers equation with the solution of the generalized KK model for $\alpha=1.1$. The logarithmic Burgers approximation captures the velocity and shape of the resulting propagating shock quite accurately as it propagates rightwise over about 100 sites. We note the presence of a small propagating bump in the exact solution which propagates on top of the shock wave and is not captured by the approximation. It forms at the front of the shock and propagates backwards, and may correspond to a solitary-like wave resulting from 
dispersive and nonlinear effects. Also, the creation of a gap and appearance of negative bead velocities are not captured by the logarithmic Burgers equation.

The case $\alpha=3 / 2$ is considered in figure 7. The first stage of the evolution of the localized perturbation displays similar features as the ones previously described, but the decay of the perturbation is faster because the initial condition is more localized (the plateau at which $\partial_{\tau} Y \approx 0$ is less broad). Despite $\epsilon \approx 0.93$ and $1-\frac{1}{\alpha}=\frac{1}{3}$ are not small, the approximation is still qualitatively correct, and even quantitatively good for capturing the shock velocity and aftershock profile. After a propagation over about 135 sites, the decay of the pulse is slightly underestimated by the logarithmic Burgers equation, with an error around $15 \%$ for relative displacements and $14 \%$ for velocities.

\section{LOGARITHMiC KdV-BuRgers Limit OF THE KUWABARA-KONO MOdEL}

5.1. Derivation of the logarithmic KdV - Burgers equation. In this section, we adapt the analysis of section 4 in order to account for dispersive effects when the dissipation constant $\gamma$ is small. As previously we assume $u \geq 0$ in (36) and we modify the Ansatz (37) as follows :

$$
u_{n}(t)=\delta y(s, \tau)^{1 / \alpha},
$$

with $s=2 \sqrt{3} \epsilon(n-c t), c=\delta^{(\alpha-1) / 2}, \tau=\sqrt{3} \epsilon^{3} c t$. The new choice of slow time scale describes the modulation due to dispersion. In what follows, we will relate the magnitudes of $\epsilon$ and $\gamma$ to the small parameter $\alpha-1$ so that nonlinear and diffusive effects act on the same time scale as dispersion.

We obtain using the chain rule and a Taylor expansion

$$
\frac{d}{d t}=c \sqrt{3}\left(\epsilon^{3} \partial_{\tau}-2 \epsilon \partial_{s}\right), \quad \Delta_{\mathrm{d}}=12\left(\epsilon^{2} \partial_{s}^{2}+\epsilon^{4} \partial_{s}^{4}\right)+O\left(\epsilon^{6}\right) .
$$

We then substitute (52) in equation (36), assume $\gamma c=O(\epsilon)$ and neglect $O\left(\epsilon^{6}\right)$ terms. After integration with respect to $s$ and some elementary algebra, one obtains

$$
\partial_{\tau}\left(y^{1 / \alpha}\right)+\frac{1}{\epsilon^{2}} \partial_{s}\left(y-y^{1 / \alpha}\right)+\partial_{s}^{3} y=c 2 \sqrt{3} \frac{\gamma}{\epsilon} \partial_{s}^{2} y
$$

with the integration constant set to 0 . This leads us to set $\epsilon=\left(1-\frac{1}{\alpha}\right)^{1 / 2}$ in (53) and to introduce

$$
\mu=c 2 \sqrt{3} \gamma\left(1-\frac{1}{\alpha}\right)^{-1 / 2} \text {. }
$$

Then we let $\alpha \rightarrow 1^{+}$in (53) under the assumption $\mu=O(1)$, which corresponds to assuming $\gamma=$ $O\left((\alpha-1)^{1 / 2}\right)$. This leads to a logarithmic KdV-Burgers equation

$$
\partial_{\tau} y+\partial_{s}(y \ln y)+\partial_{s}^{3} y=\mu \partial_{s}^{2} y
$$

in which the third spatial derivative accounts for dispersive effects.

5.2. Localized solutions of the logarithmic KdV - Burgers equation. Searching for stationary solutions $y(s)$ of (55) and integrating once with respect to $s$, we get

$$
\frac{d^{2} y}{d s^{2}}-\mu \frac{d y}{d s}+W^{\prime}(y)=0
$$

where the integration constant was set to 0 . This system corresponds to the motion of a particle in the double-well potential $W(y)=\frac{y^{2}}{2}\left(\ln |y|-\frac{1}{2}\right)$, with a saddle point at $y=0$ and two symmetric equilibria at $y= \pm 1$ (see the top left panel in figure 8). Here we are only interested in nonnegative solutions of (56) since equation (55) is defined for $y \geq 0$. Note that a continuum limit similar to (56) but with a different (polynomial) potential was also formally derived in [3].

The nontrivial equilibria are Lyapunov stable in the special case $\mu=0$ and unstable for $\mu>0$ since the particle is subjected to a negative damping. In that case, there exists an heteroclinic solution $y_{\mathrm{h}}$ of (56) such that $\lim _{s \rightarrow-\infty} y_{\mathrm{h}}(s)=1$ and $\lim _{s \rightarrow+\infty} y_{\mathrm{h}}(s)=0$. This trajectory corresponds to one side 

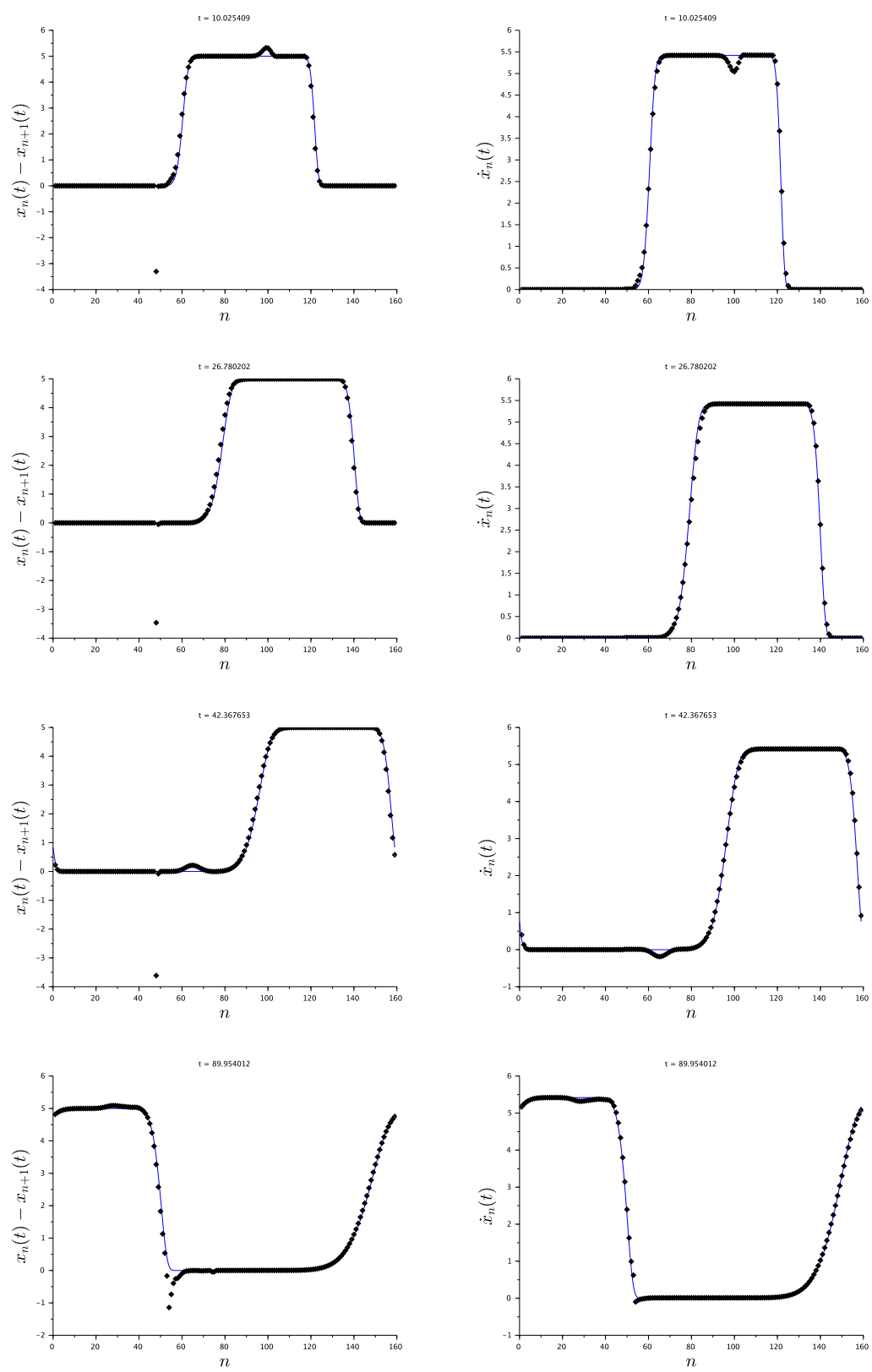

FIGURE 6. Approximate solution obtained by integrating the logarithmic Burgers equation (blue line) compared with the solution of the generalized KK model (dots). Relative displacements (left) and velocities (right) are plotted at $t \approx 10.02, t \approx 26.78$, $t \approx 42.37, t \approx 89.95$ from top to bottom. Computations are performed for $\alpha=1.1$, which corresponds to $\epsilon \approx 0.35$ and $L \approx-306$. We have fixed $\ell \approx 27.78$, $d \tilde{\xi} \approx 5.5 \times 10^{-3}, d \tau \approx 1.2 \times 10^{-5}$.

of the stable manifold of the origin (see figure 8, top right panel). To compute this solution we proceed as follows. We reformulate (56) as the first order system

$$
\frac{d y}{d s}=z, \quad \frac{d z}{d s}=\mu z-y \ln |y| .
$$



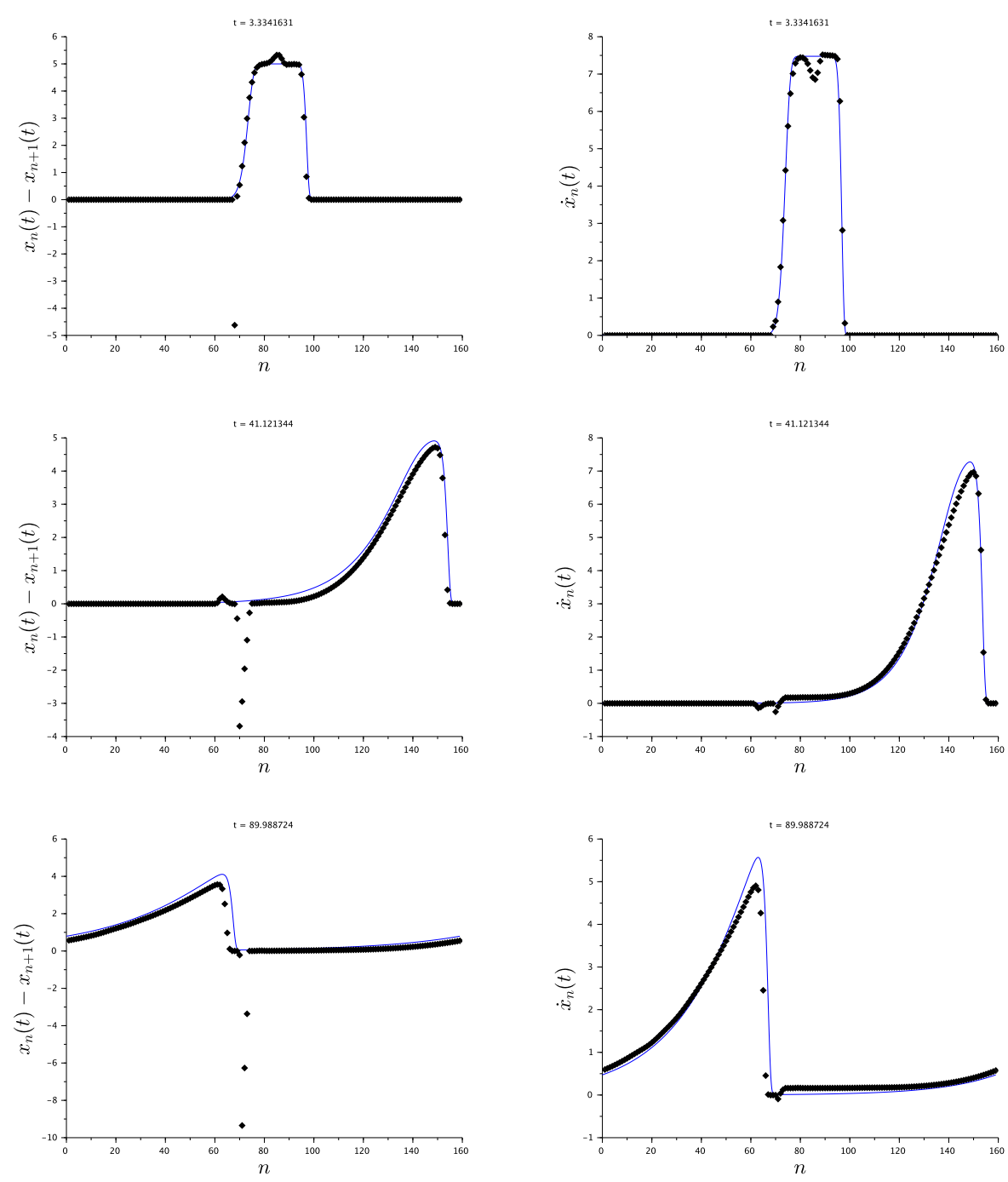

FIGURE 7. Same as in figure 6 but for $\alpha=3 / 2$. Solutions are plotted at $t \approx 3.33$, $t \approx 41.12, t \approx 90$, from top to bottom. Computations are performed with $\epsilon \approx 0.93$ and $L \approx-114.7$. We have fixed $\ell \approx 73.84, d \tilde{\xi} \approx 1.48 \times 10^{-2}, d \tau \approx 7.8 \times 10^{-5}$.

To characterize the local stable manifold of the origin in the sector $y \geq 0$ and $z \leq 0$, we set $z=$ $-\sqrt{q(y)}$ with $q \geq 0$. Using the fact that

$$
\frac{d}{d y} \frac{z^{2}}{2}=z \frac{d z}{d y}=\frac{d z}{d s}=-\mu \sqrt{q}-y \ln y
$$

the function $q=z^{2}$ satisfies

$$
\frac{d q}{d y}=2(-\mu \sqrt{q}-y \ln y)
$$

Consequently, $q$ is a nonnegative solution of the differential equation

$$
\frac{d q}{d y}=2(-\mu \operatorname{sign}(q) \sqrt{|q|}-y \ln |y|)
$$


Equation (59) is maximal monotone, hence for all initial condition $q(0)=q_{0}$ there exists a unique solution $q(y)$ defined for all $y \geq 0$ [5]. We now consider the solution $q_{\mathrm{s}}$ satisfying $q_{\mathrm{s}}(0)=0$. This solution is positive for all $y \in(0,1)$ (if not, there would be an interval where $q(y) \leq 0$ and $q^{\prime}(y) \leq 0$, which is impossible since the right side of (59) is positive for $q \leq 0$ and $y \in(0,1)$ ). Consequently, $q_{\mathrm{s}}$ defines a solution of $(58)$ for $y \in[0,1]$. Its graph corresponds to (one side of) the local stable manifold of the origin for system (57).

We compute $q_{\mathrm{s}}(y)$ numerically for $y \in[0,1]$ by integrating (59) with the initial condition $q_{\mathrm{s}}(0)=0$ (for this purpose we use the standard Scilab ODE solver). Then we obtain the heteroclinic solution $y_{\mathrm{h}}$ by integrating (forward and backward in time) equation (57) with the initial condition $\left(y_{\mathrm{h}}(0), z_{\mathrm{h}}(0)\right)=$ $\left(y_{0},-\sqrt{q_{\mathrm{s}}\left(y_{0}\right)}\right)$ for some $y_{0} \in(0,1)$. The results are illustrated in figure 8 (we have used the value $\left.y_{0}=1 / 2\right)$. When $\mu \geq 2$, we find $q_{\mathrm{s}}(1)=0$ up to machine precision, which indicates the existence of a monotonic front with $\frac{d y_{\mathrm{h}}}{d s}=z_{\mathrm{h}} \leq 0$ for all $s \in \mathbb{R}$ (see the bottom right panel in figure 8). This corresponds to the case when the unstable equilibrium $(y, z)=(1,0)$ has real eigenvalues, leading to an overdamped dynamics. For $\mu \in(0,2)$, we have an underdamped regime where $(y, z)=(1,0)$ has imaginary eigenvalues and the convergence of $\left(y_{\mathrm{h}}(s), z_{\mathrm{h}}(s)\right)$ towards $(1,0)$ when $s \rightarrow-\infty$ is oscillatory (bottom left panel in figure 8).
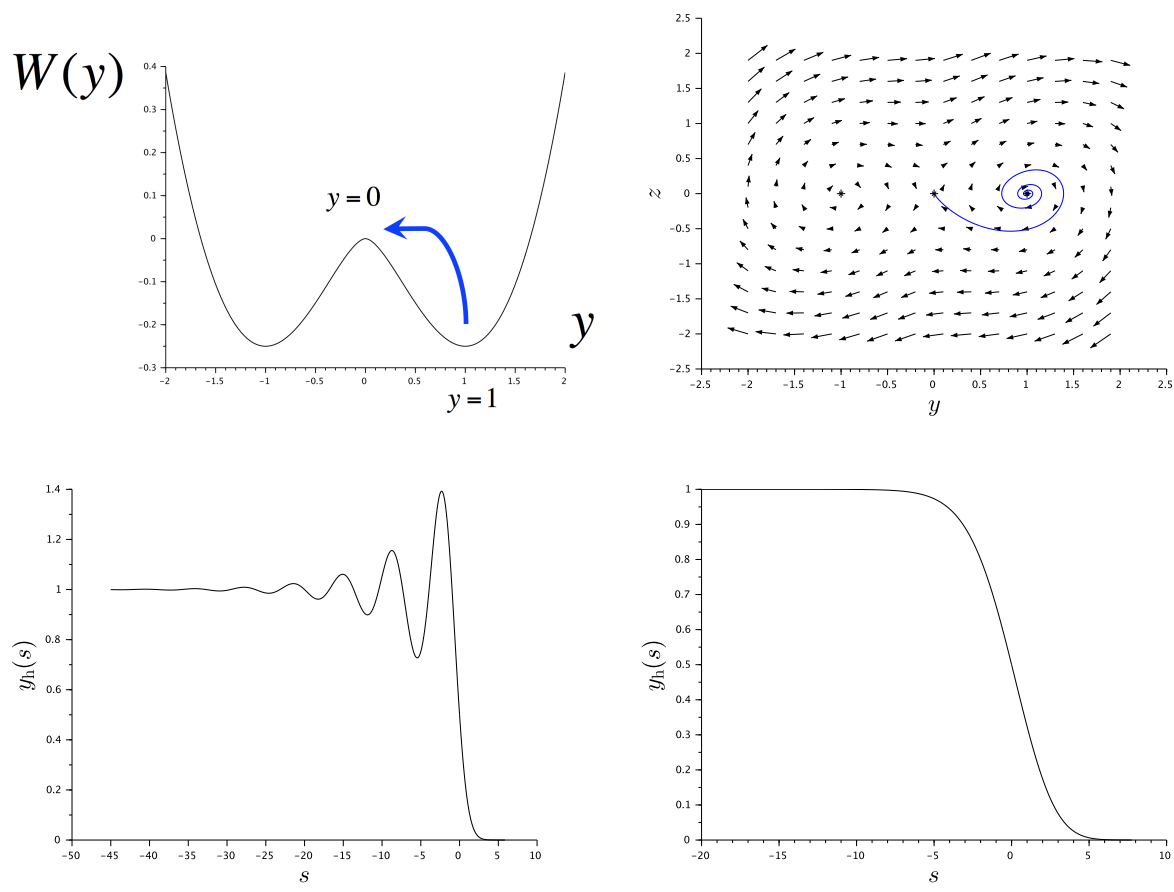

FIGURE 8. Top left : double-well potential $W$ in (56). The arrow symbolizes the heteroclinic orbit connecting the equilibrium at $y=1$ with the saddle at $y=0$ under the effect of negative damping. Top right : phase portrait of (57) for $\mu=0.3$ (underdamped regime). The blue curve corresponds to the heteroclinic orbit. Bottom : heteroclinic solutions for $\mu=0.3$ (left) and $\mu=2$ (right) which corresponds to the onset of the overdamped regime.

Remark 8. In the nondissipative case $\mu=0$, (58) has the explicit solution $q_{\mathrm{s}}(y)=-2 W(y)$. This solution corresponds to the Gaussian homoclinic solution of equation (56) with $\mu=0$, given by $y(s)=$ $\exp \left(\frac{1}{2}-\frac{s^{2}}{4}\right)[4]$. 
From Ansatz (52) we get the approximate relative displacements

$$
u_{n}(t)=\delta y_{\mathrm{h}}^{1 / \alpha}(\epsilon(n-c t)),
$$

with $\epsilon=2 \sqrt{3\left(1-\frac{1}{\alpha}\right)}, c=\delta^{(\alpha-1) / 2}$. The approximation (43) for velocities remains valid for this definition of $\epsilon$, leading to

$$
\dot{x}_{n}(t) \approx v_{\mathrm{p}}\left[y_{\mathrm{h}}-\epsilon\left(\gamma c+\frac{1}{2}\right) z_{\mathrm{h}}\right](\epsilon(n-c t)),
$$

where $v_{\mathrm{p}}=\delta^{(\alpha+1) / 2}$ is the limiting particle velocity when $n \rightarrow-\infty$. The overdamped regime occurs under the condition $\mu \geq 2$, that is

$$
\gamma \geq \sqrt{\frac{\alpha-1}{3 \alpha \delta^{\alpha-1}}}
$$

or equivalently

$$
\gamma \geq \sqrt{\frac{\alpha-1}{3 \alpha}} v_{\mathrm{p}}^{(1-\alpha) /(\alpha+1)}
$$

\section{NUMERICAL COMPUTATION OF TRAVELING FRONTS}

In this section we numerically compute traveling front solutions of the generalized KK model (35). We compare numerical solutions computed iteratively to the analytical approximations derived in sections 4 and 5, and study some of their qualitative properties.

6.1. A Gauss-Newton method for traveling fronts. We look for solutions of (35) such that $x_{n}(t)=$ $X(n-c t)$, where the profile function $X$ satisfies the boundary conditions (10) and (11) with $R_{-\infty}=$ $-\delta<0, R_{+\infty}=0$. The wave velocity is $c=\delta^{(\alpha-1) / 2}$ according to identity (21), and we recall that $\lim _{n \rightarrow-\infty} \dot{x}_{n}(t)=v_{\mathrm{p}}=\delta^{(\alpha+1) / 2}$.

Traveling fronts are obtained using a shooting method reminiscent of the one used in [3]. Let us start by describing the method for an infinite chain before considering finite chains in numerical computations. We use the dynamical formulation (31)-(32) in the case $\beta=\alpha$, and consider initial conditions $Y(0)=(x(0), w(0))$ in the affine space $\mathcal{X} \times \mathcal{Y}$ defined in section 3.2. We have then $\lim _{n \rightarrow+\infty} x_{n}(0)=0, \lim _{n \rightarrow+\infty} w_{n}(0)=0, \lim _{n \rightarrow-\infty}\left(x_{n}(0)-x_{n+1}(0)\right)=\delta$, and $\lim _{n \rightarrow-\infty} w_{n}(0)$ exists. We look for initial conditions such that the solution is defined for $t \in[0,1 / c]$ and satisfies for all $n \in \mathbb{Z}:$

$$
x_{n+1}(1 / c)=x_{n}(0), \quad w_{n+1}(1 / c)=w_{n}(0) .
$$

This condition enforces $x_{n+1}(t+1 / c)=x_{n}(t)$ by uniqueness of the solution to the initial value problem. Consequently, we get by induction $x_{n}(t)=X(n-c t)$ with $X(\xi)=x_{0}(-\xi / c)$, i.e. the solution is a traveling wave with velocity $c$. Moreover, the boundary condition (33) implies the boundary condition (11) for $X$. Since $\lim _{\xi \rightarrow-\infty} X^{\prime}(\xi)$ exists (see property (34)), the boundary condition (10) follows from (11) and the mean value theorem.

For numerical computations, we consider a finite chain with $N=421$ particles. We consider the Neumann boundary condition (25), with a free end boundary condition at $x_{N}$ (i.e. $\tilde{\delta}=0$ ) and constant compression $\delta$ at the first element.

We restrict us to initial conditions at $t=0$ taking the form

$$
Y(0)=\left(x_{1}(0), \ldots, x_{n_{0}-1}(0), x_{n_{0}+1}(0)+\frac{\delta}{2}, x_{n_{0}+1}(0), \ldots, x_{N-1}(0), 0, w_{1}(0), \ldots, w_{N}(0)\right),
$$

where $n_{0}=(N+1) / 2=211$. Indeed, imposing $x_{n_{0}}(0)-x_{n_{0}+1}(0)=\frac{\delta}{2}$ allows one to fix the phase of the front, and $x_{N}(0)=0$ fixes the translation of the chain. In order to solve the nonlinear system 
(64), we look for an initial condition that minimizes the function of $2 N-2$ variables

$$
\mathcal{S}=\sum_{n=1}^{N}\left(x_{n+1}(1 / c)-x_{n}(0)\right)^{2}+\left(w_{n+1}(1 / c)-w_{n}(0)\right)^{2},
$$

in which we fix $x_{N+1}(1 / c):=x_{N}(1 / c), w_{N+1}(1 / c):=w_{N}(1 / c)$. The minimization of $\mathcal{S}$ is realized using the Gauss-Newton method [2] (in [3], a fixed point iteration was used to solve the nonlinear system (64)).

The iterative method is initialized using a kink-type Ansatz which is scaled according to the KdVBurgers limit described in section 5. More precisely, we consider the initial relative displacements $u_{n}(0)=x_{n}(0)-x_{n+1}(0)=U\left(n-n_{0}\right)$ with

$$
U(\xi)=\frac{\delta}{2}(1-\tanh (\lambda \xi)), \quad \lambda=2 \sqrt{3}\left(1-\frac{1}{\alpha}\right)^{1 / 2},
$$

and the initial relative velocities $\dot{u}_{n}(0)=-c U^{\prime}\left(n-n_{0}\right)$ given by

$$
\dot{u}_{n}(0)=\frac{\delta^{(\alpha+1) / 2}}{2} \lambda\left(1-\tanh ^{2}\left(\lambda\left(n-n_{0}\right)\right)\right) .
$$

This choice determines the initial displacements by induction through the formula

$$
x_{N}(0)=0, \quad x_{n}(0)=x_{n+1}(0)+u_{n}(0) \text { for } n=N-1, \ldots, 1,
$$

and the initial velocities through

$$
\dot{x}_{N}(0)=\dot{u}_{N}(0), \quad \dot{x}_{n}(0)=\dot{x}_{n+1}(0)+\dot{u}_{n}(0) \text { for } n=N-1, \ldots, 1,
$$

which in turn provides the generalized initial velocities $w_{n}(0)$ using formula (31).

The Gauss-Newton iterations are stopped when $\mathcal{S}$ drops below $10^{-14}$ and each components of two successive iterates differ at most by $10^{-7}$. We have observed that the accuracy of the initial seed is not very important for the convergence of the iteration, but the evaluation of the Jacobian matrix in the Gauss-Newton method needs to be precise (for this purpose we use the numderivative function of the software package Scilab).

Once the iteration has converged and the resulting initial condition $Y(0)=(x(0), w(0))$ is integrated for $t \in[0,1 / c)$, one obtains $X(\xi)=x_{n}(t)$ and $X^{\prime}(\xi)=-\frac{\dot{x}_{n}(t)}{c}$ for $\xi=n-c t \in(n-1, n]$ and for all $n=1, \ldots, N$.

To check the numerical method, we have integrated the computed initial conditions over long times for different values of parameters $\alpha, \delta, \gamma$. We have observed a steady propagation of the traveling front over long distances, as illustrated in figure 9.

6.2. Qualitative properties of fronts. When $\gamma$ lies below a critical value $\gamma_{c}$, we observe that the front is non-monotonic within the compressed region. More precisely, the $\operatorname{limits} \lim _{n \rightarrow-\infty} \dot{x}_{n}(t)=$ $\lim _{t \rightarrow+\infty} \dot{x}_{n}(t)=v_{\mathrm{p}}$ and $\lim _{n \rightarrow-\infty}\left(x_{n}(t)-x_{n+1}(t)\right)=\lim _{t \rightarrow+\infty}\left(x_{n}(t)-x_{n+1}(t)\right)=\delta$ occur with oscillations around the limiting values, as illustrated in the left panel of figure 9 . This case is referred to as the underdamped regime. On the contrary, in the overdamped regime $\gamma \geq \gamma_{c}$, we obtain monotonic fronts as illustrated in the right panel of figure 9. More precisely, we observe some numerical oscillations of velocities and relative displacements around the limiting values at $n=-\infty$, but their amplitude is negligible (it is close to $10^{-8}$ or below, i.e. smaller than the numerical precision of the iterative method).

The critical damping takes the form

$$
\gamma_{c}=\delta^{(1-\alpha) / 2} \Gamma(\alpha)
$$

where the multiplicative constant $\Gamma(\alpha)$ needs to be determined. The form (66) can be obtained by rescaling (35) and the front solution as described in section 3.1, which yields (similarly to (30)) a dimensionless damping parameter $\gamma \delta^{(\alpha-1) / 2}$ with critical value denoted by $\Gamma(\alpha)$. 

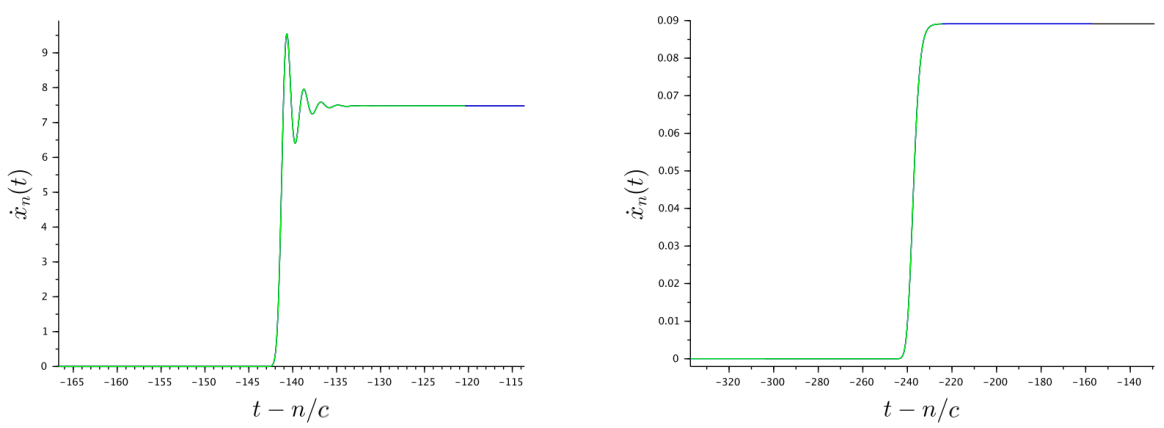

FIGURE 9. Graph of bead velocities versus $t-n / c$ for $n=271$ (black line), $n=331$ (blue line), $n=391$ (green line), for the initial condition computed with the GaussNewton method. The three graphs coincide almost perfectly. Left plot : $\alpha=3 / 2$, $\delta=5, \gamma=0.06$. Right plot : $\alpha=1.1, \delta=0.1, \gamma=0.2$.

One can notice that $\gamma_{c}$ diverges when the compression $\delta$ at $n=-\infty$ goes to 0 . Consequently, once $\alpha>1$ and $\gamma$ are fixed, the underdamped regime $\gamma<\gamma_{c}$ occurs when the compression $\delta$ is small enough, or equivalently when the asymptotic bead velocity $v_{\mathrm{p}}$ at $n=-\infty$ is small. This feature of the generalized KK model contrasts with the properties of generalized Hertzian chains with linear dashpots $(\alpha>1, \beta=1)$ for which $\gamma_{c}$ vanishes with $\delta$ [19]. For example, in the numerical simulations performed in [36] for $\alpha=3 / 2$, the underdamped regime occurs when $v_{\mathrm{p}}$ is large enough.

We observe numerically that the multiplicative constant $\Gamma(\alpha)$ in (66) becomes small when $\alpha$ approaches unity. This is consistent with the analytical approximation (62) which indicates that

$$
\Gamma(\alpha) \approx \sqrt{\frac{\alpha-1}{3 \alpha}} \text { for } \alpha \approx 1^{+} .
$$

This phenomenon is illustrated for $\alpha=1.02$ and $\delta=1$ in figure 10, which compares the maximal bead velocity $\|\dot{x}\|_{\infty}$ to the downstream particle velocity $v_{\mathrm{p}}$. The transition between the underdamped and overdamped regimes occurs at $\gamma_{c} \approx 0.079$, a value well approximated by (63), which yields a critical damping close to 0.081. Of course the approximation (62) derived for $\alpha \rightarrow 1^{+}$deteriorates when $\alpha$ increases. For example, numerical computations indicate $\gamma_{c} \approx 0.26$ for $\alpha=3 / 2$ and $\delta=1$ (see figure 11 ), whereas the analytical approximation (63) yields a higher critical damping equal to $1 / 3$.

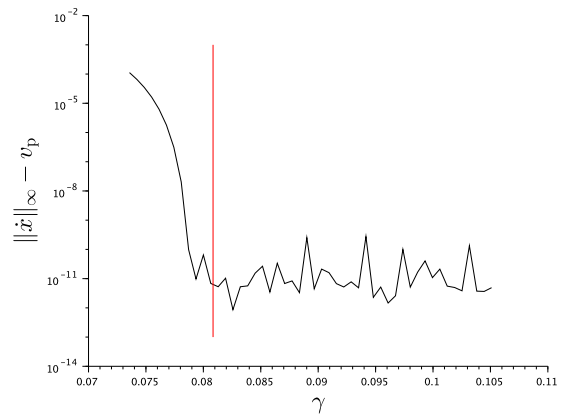

FIGURE 10. Graph of $\|\dot{x}\|_{\infty}-v_{\mathrm{p}}$ versus $\gamma$ for $\alpha=1.02$ and $\delta=1$. One observes a transition between the underdamped and overdamped regimes at $\gamma_{c} \approx 0.079$, close to the analytical approximation (63) (red line). 
An important feature of the underdamped regime is that the relative difference (overstrike) between the maximal bead velocity (or relative displacement) and the limit at $n=-\infty$ imposed by the boundary condition can be significant. This phenomenon is illustrated in figure 11 for $\alpha=3 / 2$ and $\delta=1$. Below $\gamma=\gamma_{c}$, we observe that the overstrike increases with decreasing $\gamma$, and becomes close to 1.5 for $\gamma=0.03$. For smaller values of $\gamma$, the iterative method does not reach the error bounds given above, so we cannot conclude on the limiting behavior of the front profile. These numerical difficulties are consistent with the fact that traveling fronts (with a constant compression at $n=-\infty$ ) do not exist in the limit case $\gamma=0$ (see remark 4).
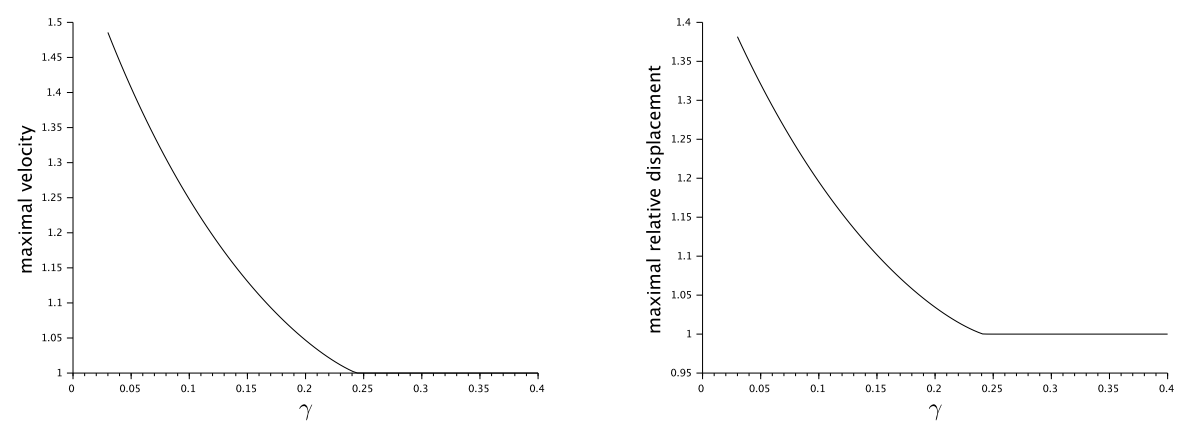

FigURE 11. Left : graph of $\|\dot{x}\|_{\infty}$ versus $\gamma$ for $\alpha=3 / 2$ and $\delta=1$. Right : graph of $\|u\|_{\infty}$ versus $\gamma$, where $u_{n}=x_{n}-x_{n+1}$ corresponds to relative displacements. One observes a transition between the underdamped and overdamped regimes at $\gamma_{c} \approx 0.26$.

6.3. Comparison with the continuum limits. In sections 4 and 5 , we have derived two different continuum limits of the generalized KK model when $\alpha \rightarrow 1^{+}$. The logarithmic Burgers equation was derived for $\gamma \delta^{(\alpha-1) / 2} \gg\left(1-\frac{1}{\alpha}\right)^{1 / 2}$, and admits explicit front solutions with monotonic relative displacement profiles. The logarithmic KdV-Burgers equation was obtained for a weak dissipation with $\gamma \delta^{(\alpha-1) / 2}=O\left(1-\frac{1}{\alpha}\right)^{1 / 2}$. Depending whether the rescaled dissipation constant $\mu$ defined in (54) lies below or above 2 , it leads to underdamped or overdamped fronts. The front profiles must be computed numerically, but at a cost well below the complexity of the direct approach of section 6.1.

Figure 12 illustrates the convergence of the analytical approximations when $\alpha$ approaches unity, for $\delta=5$ and $\alpha=1.02$. In the left panel, we fix $\gamma=0.2$ (overdamped regime) and compare the numerical front solution obtained with the Gauss-Newton method to the logarithmic Burgers approximation. In the right panel we fix $\gamma=0.06$ (underdamped regime, $\mu \approx 1.51$ ) and compare the numerical front solution to the logarithmic KdV-Burgers approximation. The graphs of the analytical and numerical solutions are almost identical in both case.

Next we study how the two analytical approximations compare with the numerical solution when we depart from their asymptotical range of validity, i.e. when $\alpha$ is further increased. For this purpose we fix $\delta=5$ and first consider the case $\alpha=1$.1. In figure 13, we fix $\gamma=0.2$ (leading to the overdamped regime) and compare the numerical solution with the logarithmic Burgers approximation. We find an excellent agreement both for relative displacements and velocities. In figure 14, we set $\gamma=0.06$ (leading to the underdamped regime) and compare the numerical solution with the logarithmic KdVBurgers approximation. The approximation is excellent for relative displacements (left panel) but small discrepancies appear in the approximation of velocities, for which the overstrike is overestimated by $2.7 \%$ (green curve in the right panel). It is also interesting to observe the effect of the first order correction in $\epsilon$ which is taken into account in (61) for the computation of the green curve. When this term is neglected (red curve), the front width is overestimated by around $11.5 \%$ (but the overstrike is closer to the value obtained with the numerical solution). 

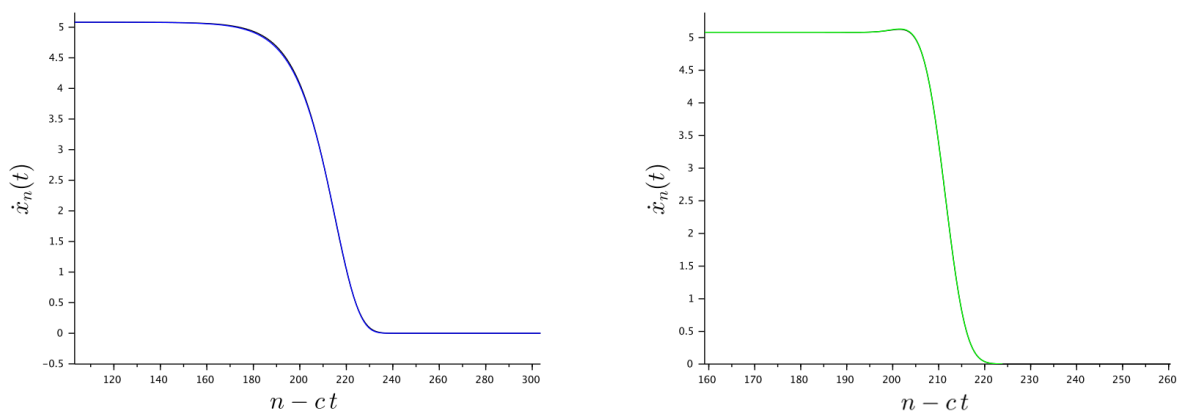

FIGURE 12. Comparison of the traveling front computed with the Gauss-Newton method (black line), the approximation (48) derived from the logarithmic Burgers equation (left plot, blue line), and the approximation (61) obtained with the logarithmic KdV-Burgers equations (right plot, green line). The numerical solutions and analytical approximations are almost identical. We only represent bead velocities (relative displacements display similar features). We have fixed $\delta=5$ and $\alpha=1.02$. The left panel corresponds to $\gamma=0.2$ and the right panel to $\gamma=0.06$.
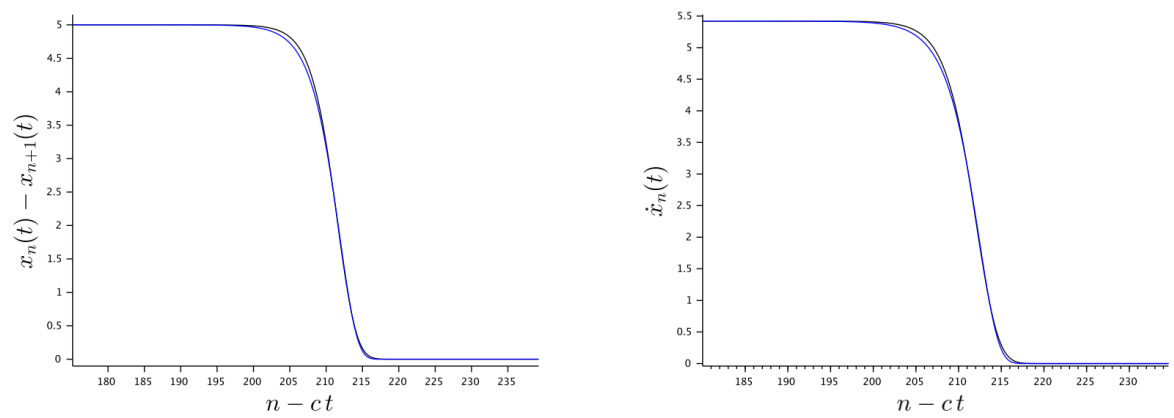

FIGURE 13. Comparison of the traveling front computed with the Gauss-Newton method (black line) and the approximations (47) and (48) derived from the logarithmic Burgers equation (blue line). The left panel corresponds to relative displacements and right panel to velocities. We have fixed $\delta=5, \alpha=1.1, \gamma=0.2$.

We now further increase $\alpha$ to the classical value $\alpha=1.5$. Figure 15 compares the analytical approximations derived from the logarithmic Burgers and KdV-Burgers equations with the traveling fronts computed iteratively. For $\gamma=0.2$ (overdamped regime), the velocity profile obtained with the logarithmic Burgers approximation is quite close to the numerical solution (top right plot, blue curve). The logarithmic Burgers approximation of relative displacements shows more discrepancies (top left plot, blue curve) but the approximation remains meaningful. As noted in section 6.2, the critical value of the damping is overestimated by the logarithmic KdV-Burgers approximation. As a consequence, the associated approximation of the front is underdamped $(\mu \approx 1.8)$, leading to a spurious overstrike in the approximation of the velocity (top right plot, green curve). However, the overstrike is negligible for relative displacements (top left plot, green curve). As a conclusion, the logarithmic KdV-Burgers approximation displays clear discrepancies with the numerical solution but remains meaningful.

The bottom plots in figure 15 correspond to $\gamma=0.06$ (underdamped regime). Here the logarithmic Burgers approximation is not appropriate, since it does not capture the rather large oscillations of relative displacements around the compressed state (bottom left plot, blue curve). However, on can notice 

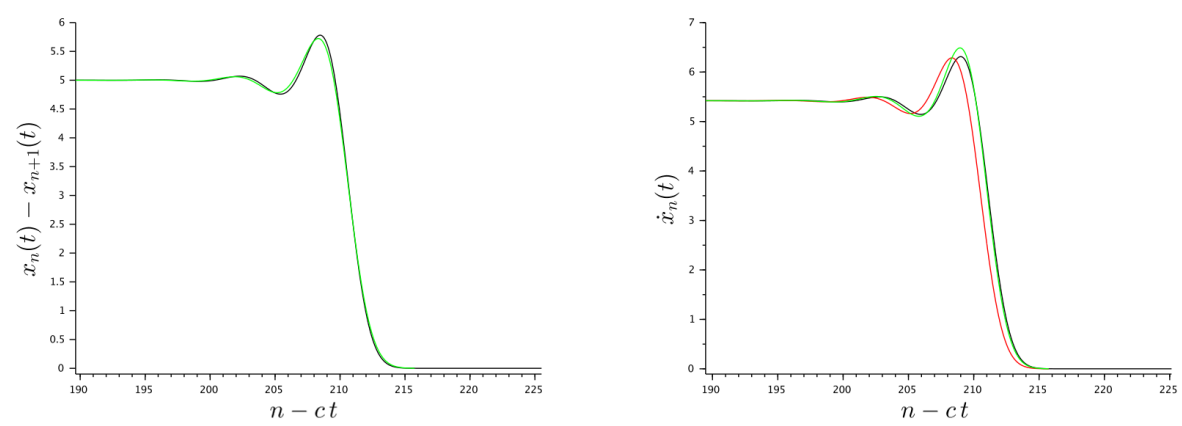

FIGURE 14. Comparison of the traveling front computed with the Gauss-Newton method (black line) and the approximations (60) and (61) obtained with the logarithmic KdV-Burgers equations (green line). The red line corresponds to the $O\left(\epsilon^{0}\right)$ order terms in approximation (61). The left panel corresponds to relative displacements and right panel to velocities. We have fixed $\delta=5, \alpha=1.1, \gamma=0.06$.

that the correction term in the velocity approximation (48) accounts for an overstrike (bottom right plot, blue curve). The logarithmic KdV-Burgers approximation (bottom plots, green curves) displays reasonable agreement with the numerical solution. The approximation of the shock width is quite accurate and the period of oscillations at the top of the front is reasonably approximated. The overstrike in relative displacements is underestimated by around $5 \%$, and the overstrike in velocity is overestimated by around $14 \%$. This error can be reduced to $2 \%$ by considering only the zeroth order term in $\epsilon$ in (61), but then the relative error on the front width is multiplied by two (data not shown).

\section{CONCLUSION AND PERSPECTIVES}

In this work, we have analyzed the propagation of fronts in one-dimensional lattices including both dissipative and nonlinear elastic nearest neighbors interactions. In section 2, we have determined in the general case how the velocity of traveling fronts depends on the asymptotic states of the system at $\pm \infty$. In section 3, we have considered a lattice model for viscoelastic granular chains that falls within the above class, in particular the KK model with Hertzian elastic and dissipation potentials, and a generalized KK model in which the exponent $\alpha$ in the contact force is an additional parameter. We have studied some qualitative properties of the models, and we have shown numerically that fronts can be generated in the KK model by applying a constant pressure at one end of a chain initially at rest. In the limit when $\alpha$ is close to unity, we have formally derived two different amplitude equations for long waves, namely a logarithmic Burgers equation (section 4) and a logarithmic KdV-Burgers equation (section 5). The latter incorporates dispersive effects that must be taken into account when contact damping is small. Both models possess traveling front solutions that have been compared to direct numerical computations of fronts in the granular chain based on the Gauss-Newton method (section 6). We have observed the convergence of the analytical approximations towards numerical solutions when $\alpha$ goes to unity, and we have checked that the analytical approximations still provide meaningful results when $\alpha$ is increased up to the classical value $3 / 2$. In addition, we have found a transition from underdamped to overdamped fronts when a nondimensional damping parameter $\gamma$ lies above some threshold $\gamma_{\mathrm{c}}$. Using the logarithmic KdV-Burgers approximation, we have obtained an approximation of $\gamma_{\mathrm{c}}$ valid for $\alpha$ close to unity.

Several significant extensions of this work could be considered. From a theoretical point of view, it would be interesting to justify the amplitude equations and analytical approximations derived in sections 4 and 5. In particular, we have observed in section 4.3 that the logarithmic Burgers equation is able 

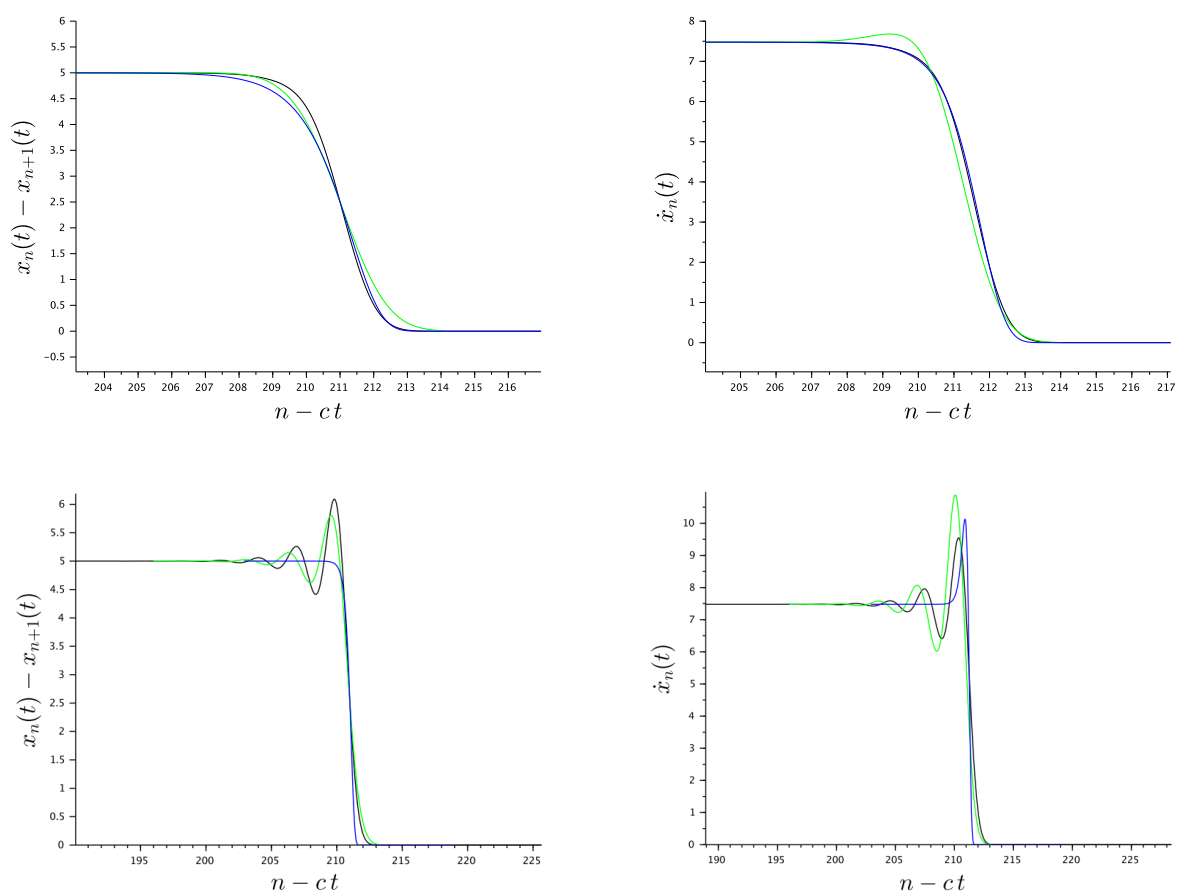

FIGURE 15. Comparison of the traveling front computed with the Gauss-Newton method (black line), the approximations (47) and (48) derived from the logarithmic Burgers equation (blue line), and the approximations (60) and (61) obtained with the logarithmic KdV-Burgers equations (green line). Left panels correspond to relative displacements and right panels to velocities. We have fixed $\delta=5, \alpha=1.5$. Top plots : $\gamma=0.2$, and bottom plots : $\gamma=0.06$.

to approximate the evolution over long times of well-prepared localized initial conditions when $\alpha$ is close to unity. It would be interesting to derive error bounds on the logarithmic Burgers approximation valid over such time scales, and to perform the same type of analysis for the logarithmic KdV-Burgers equation, in the same spirit as previous justifications of modulation equations for precompressed granular chains (see [11] and references therein). Another interesting open problem is to prove the existence and stability of exact traveling fronts in the granular chain, in particular with profiles close to the logarithmic Burgers or KdV-Burgers approximations in appropriate parameter regimes. In this context, the analytical approximations obtained in this work could provide a good starting point to construct exact front solutions using a fixed point technique. From a numerical point of view, it would be worthwhile to study the qualitative properties of fronts for more general values of the exponent $\beta$ in (2) and to take into account the energy dissipation due to plasticity.

Acknowledgement. The author is grateful to Bernard Brogliato for stimulating discussions.

\section{REFERENCES}

[1] Allaire G 2007 Numerical Analysis and Optimization. An introduction to mathematical modelling and numerical simulation Numerical Mathematics and Scientific Computation, Oxford Science Publications (New York: Oxford University Press Inc.)

[2] Argyros I K, Cho Y J and Hilout S 2012 Numerical methods for equations and its applications (CRC Press)

[3] Aubry S and Proville L 2007 Pressure fronts in $1 D$ nonlinear lattices, to appear in Proceedings of EQUADIFF-07, arXiv:0910.4890 
[4] Bialynicki-Birula I and Mycielski J 1976 Nonlinear wave mechanics Annals of Physics $10062-93$

[5] Brézis H 1973 Opérateurs maximaux monotones et semi-groupes de contractions dans les espaces de Hilbert Mathematics Studies 5 (North-Holland)

[6] Brilliantov N V, Pimenova A V and Goldobin D S 2015 A dissipative force between colliding viscoelastic bodies: Rigorous approach EPL 10914005

[7] Brogliato B 2016 Nonsmooth Mechanics: Models, Dynamics and Control Communications and Control Engineering. Third Edition (Switzerland: Springer International Publishing)

[8] Burgoyne H A, Newman J A, Jackson W C and Daraio C 2015 Guided impact mitigation in 2D and 3D granular crystals Procedia Engineering $\mathbf{1 0 3} 52-59$

[9] Carretero-González R, Khatri D, Porter M A, Kevrekidis P G and Daraio C 2009 Dissipative solitary waves in granular crystals Phys. Rev. Lett. 102024102

[10] Chatterjee A 1999 Asymptotic solution for solitary waves in a chain of elastic spheres Phys. Rev. E 59 5912-5919

[11] Chong C, Kevrekidis P G and Schneider G 2014 Justification of leading order quasicontinuum approximations of strongly nonlinear lattices Disc. Cont. Dyn. Sys. A 34 3403-3418

[12] Duvall G E , Maniv R and Lowell S C 1969 Steady shock profile in a one-dimensional lattice J. Appl. Phys. 40 37713775

[13] Falcon E 1997 Comportements dynamiques associés au contact de Hertz : processus collectifs de collision et propagation d'ondes solitaires dans les milieux granulaires, $\mathrm{PhD}$ thesis, Université Claude Bernard Lyon 1

[14] Falcon E, Laroche C, Fauve S and Coste C 1998 Behavior of one inelastic ball bouncing repeatedly off the ground Eur. Phys. J. B 3 45-57

[15] Falcon E, Laroche C, Fauve S and Coste C 1998 Collision of a 1-D column of beads with a wall Eur. Phys. J. B 5 111-131

[16] Fermi E, Pasta J and Ulam S 1955 Studies of nonlinear problems Technical Report LA-1940, Los Alamos National Laboratory

[17] Fraternali F, Porter M A and Daraio C 2010 Optimal design of composite granular protectors Mech. Adv. Mat. Struct. $171-19$

[18] Goldobin D S, Susloparov E A, Pimenova A V and Brilliantov N V 2015 Collision of viscoelastic bodies: Rigorous derivation of dissipative force Eur. Phys. J. E 3855

[19] Herbold E B and Nesterenko V F 2007 Shock wave structure in a strongly nonlinear lattice with viscous dissipation Phys. Rev. E 75021304

[20] Herrmann M and Rademacher J D M 2010 Riemann solvers and undercompressive shocks of convex FPU chains Nonlinearity 23 277-304

[21] Herrmann M and Rademacher J D M 2010 Heteroclinic travelling waves in convex FPU-type chains SIAM J. Math. Anal. 42 1483-1504

[22] Herrmann M 2011 Action minimizing fronts in general FPU-type chains J. Nonlinear Sci. 21 33-55

[23] Hietarinta J, Kuusela T and Malomed B A 1995 Shock waves in the dissipative Toda lattice J. Phys. A: Math. Gen. 28 3015-3024

[24] Hinch E J and Saint-Jean S 1999 The fragmentation of a line of balls by an impact. Proc. R. Soc. Lond. A 455, 3201-3220

[25] Hunt K H and Crossley F E 1975 Coefficient of restitution interpreted as damping in vibroimpact Journal of Applied Mechanics 42 440-445

[26] Iooss G 2000 Travelling waves in the Fermi-Pasta-Ulam lattice Nonlinearity 13 849-866

[27] James G and Pelinovsky D 2014 Gaussian solitary waves and compactons in Fermi-Pasta-Ulam lattices with Hertzian potentials Proc. R. Soc. A 470 (2165) 20130462

[28] James G and Starosvetsky Y 2014 Breather solutions of the discrete $p$-Schrödinger equation, in Localized Excitations in Nonlinear Complex Systems, Eds. Carretero-González R, Cuevas-Maraver J, Frantzeskakis D, Karachalios N, Kevrekidis P G , Palmero-Acebedo F Nonlinear Systems and Complexity 7 77-115 (Springer)

[29] James G 2018 Travelling breathers and solitary waves in strongly nonlinear lattices Phil. Trans. R. Soc. A 37620170138

[30] James G, Vorotnikov K and Brogliato B 2020 Kuwabara-Kono numerical dissipation: a new method to simulate granular matter IMA Journal of Applied Mathematics $\mathbf{8 5} 27-66$

[31] Jayaprakash K R, Starosvetsky Y and Vakakis A F 2011 New family of solitary waves in granular dimer chains with no precompression Phys. Rev. E 86, 036606

[32] Johnson K L 1985 Contact Mechanics (Cambridge: Cambridge Univ. Press)

[33] Karpman V 1975 Nonlinear waves in dispersive media (New York: Pergamon)

[34] Khatri D, Ngo D and Daraio C 2012 Highly nonlinear solitary waves in chains of cylindrical particles Granular Matter 14 63-69

[35] Kuwabara G and Kono K 1987 Restitution coefficient in a collision between two spheres Japanese Journal of Applied Physics 26 1230-1233 
[36] Liang T, Zhu X L, Meng Z C, Duan W S and Yang L 2019 Shock waves in nonlinear granular chain with viscosity under Hertz contact J. Phys. Soc. Jpn. 88, 034002

[37] Luding S, Clément E, Blumen A, Rajchenbach J and Duran J 1994 Anomalous energy dissipation in molecular-dynamics simulations of grains: The "detachment" effect Phys. Rev. E $504113-4122$

[38] Mielke A and Patz C 2010 Dispersive stability of infinite-dimensional Hamiltonian systems on lattices Applicable Analysis 89 1493-1512

[39] Molinari A and Daraio C 2009 Stationary shocks in periodic highly nonlinear granular chains Phys. Rev. E 80056602

[40] Nesterenko V F 2001 Dynamics of Heterogeneous Materials (Berlin, Germany: Springer)

[41] Nesterenko V F 2003 Shock (blast) mitigation by "soft" condensed matter. In Granular Material-Based Technologies, Proc. MRS Symp., 759 (eds Sen S, Hunt M L, Hurd A J), pp. MM4.3.1-4.3.12 (Pittsburgh, PA: MRS Press) doi:10.1557/PROC-759-MM4.3

[42] Ngo D, Griffiths S, Khatri D and Daraio C 2013 Highly nonlinear solitary waves in chains of hollow spherical particles Granular Matter 15 149-155

[43] Nguyen N S and Brogliato B 2014 Multiple Impacts in Dissipative Granular Chains Lecture Notes in Applied and Computational Mechanics 72 (Berlin, Germany: Springer)

[44] Porter M, Kevrekidis P G and Daraio C 2015 Granular crystals : Nonlinear dynamics meets materials engineering Physics Today $\mathbf{6 8} 44$

[45] Ramírez R, Pöschel T, Brilliantov N V and Schwager T 1999 Coefficient of restitution of colliding viscoelastic spheres Phys. Rev. E 60 4465-4472

[46] Ricci L, Nguyen V H, Sab K, Duhamel D and Schmitt L 2005 Dynamic behaviour of ballasted railway tracks: A discrete/continuous approach Computers and Structures $\mathbf{8 3} 2282-2292$

[47] Rosenau P 2007 Compactification of patterns by a singular convection or stress Phys. Rev. Lett. 99234102

[48] Sekimoto K 2010 Newton's cradle versus nonbinary collisions Phys. Rev. Lett. 104124302

[49] Sen S, Hong J, Bang J, Avalos E and Doney R 2008 Solitary waves in the granular chain Physics Reports 462 21-66

[50] Simon R 1967 The development of a mathematical tool for evaluating golf club performance Proceedings of ASME Design Engineering Conference, New York city, USA.

[51] Sun D, Daraio C and Sen S 2011 Nonlinear repulsive force between two solids with axial symmetry Phys. Rev. E 83 066605

[52] Sun D and Sen S 2013 Nonlinear grain-grain forces and the width of the solitary wave in granular chains : a numerical study Granular Matter 15 157-161

[53] Starosvetsky Y, Jayaprakash K R, Hasan M A and Vakakis A F 2017 Topics on the Nonlinear Dynamics and Acoustics of Ordered Granular Media (Singapore: World Scientific)

[54] Yasuda H, Chong C, Yang J and Kevrekidis P G 2017 Shock and rarefaction waves in generalized Hertzian contact models Phys. Rev. E 95062216

${ }^{1}$ InRIA Grenoble - Rhône-Alpes, Tripop TeAm, Inovallée, 655 AVenue de l'Europe, 38334 SAInt Ismier Cedex, France, ${ }^{2}$ Univ. Grenoble Alpes, CNRS, Inria, Grenoble INP*, LJK, 38000 Grenoble, France. * Institute of ENGINEERING Univ. GRENOble AlPES

E-mail address: guillaume. james@inria.fr 